\title{
MEMBERSHIP LISTING
}

$-\mathrm{A}-$

RUTH L. AASKOV, DLL, Augsburg College, Minneapolis, Minn. 55404 DOLORES ABALLI, DLL, St. Mary's College, Notre Dame, Ind. 46556 ACQUISITIONS LIBRARIAN, North Texas State University, Library Department, Denton, Texas 76203

RICHARD ADAM, DLL, Western Reserve Academy, Hudson, Ohio 44236

KENNETH R. ADAMS, Weber State College, Foreign Language Department, Ogden, Utah 84403

MRS. SUDIE M. ADAMS, Asst. Professor, Spanish and Portuguese, Baylor University, Waco, Texas 76703

CHARLES ADAMSON, 821 Osceola Avenue, St. Paul, Minn. 55105

DWAYNE ADCOCK, Coord. FL, Eugene Public Schools, 200 North Monroe St., Eugene, Oregon 97402

JEAN ADJEMIAN, 430 Buffalo Avenue, Niagara Falls, N. Y. 14303

ROLENA K. ADORNO, Director, University of Hartford, 200 Bloomfield Avenue, Hartford, Ct. 06617

NELLIE AGOSTINO, Chmn. Lang. Dept., East Hart High School, 777 Burn. side Avenue, E. Hartford, Ct. 06117

A. A. AGUIRRE, Span. Dept. Head, Indiana University at South Bend, Northside Blvd., South Bend, Ind. 46615

MRS. OLGA AGUIRRE, DLL, Stratford College, Danville, Va. 24541

JOHN A. AHREN, Westchester Community College, 75 Grasslands Rd. Valhalla, N. Y. 10595

CHRISTA AHRENS, DLL, Georgia State College, P. O. Box 415, Atlanta, Ga. 30303

H. F. AIKENS, DLL, Dalhonisie University, Halifax, Nova Scotia, Canada

FAROUK B. ALAMEDDINE, DLL, Wayne State University, 20023 Trinity Avenue, Detroit, Mich. 48219

UNIVERSITY OF ALBERTA, Romance Lang./Lang. Lab, Edmonton, Alberta, Canada

KENT ALCORN, 7723 Chambers Creek Road, Tacoma, Wash. 98467

REBECCA A. ALDRIDGE, Northern Oklahoma College, 1008 E. Oklahoma, Tonkawa, Okla. 74653

VIRGINIA BROWN ALIOTTI, DLL, Madison College, Box X, Harrisonburg, Va. 22801

DR. HAL ALLEN, Assoc. Professor of German, Hendrix College, Conway, Ark. 72032

ROBERT F. ALLEN, DLL, Rutgers University, 508 Newark Avenue, Piscataway, N. J. 08854

LEE (LEON) ALTMAN, DLL, San Francisco State College, 1600 Holloway, San Francisco, Cal. 94132

GARY JOHN AMBERT, DLL, E. Carolina University, Greenville, N. C. 27834

SR. ROSE TERESA AMOR, Chmn., Spanish Department, Molloy Catholic College, Rockville CTP, New York, N. Y. 11570

CHMN. DEPT. MOD. LANG., Andrews University, Berrien Springs, Mich. 49104

PATRICIA C. ANNABEL, Slippery Rock State College, Slippery Rock, Pa. 16057

NAZMI ARANITI, Quinnipiac College, 303 Noble St., West Haven, Ct. 06516 
N. D. ARBAIZA, Chmn., Department of Foreign Languages, Rockland Community College, 145 College Road, Suffern, N. Y. 10901

J. ARENDT, Minneapolis Schools, 3709 48th Avenue South, Minneapolis, Minn. 55406

IMOGENE C. ARENSMAN, Metropolis Community High School, 1004 Catherine Street, Metropolis, Ill. 62960

ARIZONA STATE UNIVERSITY, Library Periodicals, Tempe, Ariz. 85281 JOANNE ARMSTRONG, Assoc. Professor, University of Texas at El Paso, Box 5, El Paso, Tex. 79902

KATHRYN ARNOLD, DLL, Adelphi University, Garden City, N. Y. 11530

F. C. ASH, DLL, 3904 Vardell Road N. W., Calgary, Alta, Canada

GUY ASSELIN, DLL, Carleton University, Ottawa 1, Ontario, Canada

CECIL E. ATTIG, California State College, 800 N. State College Blvd., Fullerton, California

PROFESSOR G. E. AUCOIN, Box 760, Sydney, Nova Scotia, Canada

THE LIBRARIAN, Auckland Teachers College, 74 Epson Avenue, Auckland, SE 3, N. Zealand

AUDIO-VISUALS LIBRARY, G-20 Hillman Library, University of Pittsburgh, Pittsburgh, Pa. 15213

GILLES E. AUGER, Nasson College, Springvale, Maine 04083

SR. MARY AUGUSTA, Professor, French and Spanish, Xavier University of Louisiana, Pine and Palmetto Streets, New Orleans, Louisiana 70125

ROBERT P. AUSTIN, DLL, Loyola Academy, 1100 N. Laramie, Wilmette, Illinois 60091

DR. L. E. BAAK, Morningside College, Sioux City, Iowa 51106

J. W. BABIN, Assoc. Professor of French, St. Francis Xavier University, Antigonish, Nova Scotia, Canada

MARY BAECHLE, C. L. Community High School, 45 W. Franklin St., Crystal Lake, Ill. 60014

JOHN C. BAIMAS, Fitchburg High School, Fitchburg, Mass. 01420

REID E. BAKER, 2040 Iuka Avenue, Columbus, Ohio 43201

ROBERT L. BAKER, Chmn., Dept. of Russian, Middlebury College, Middlebury, Vt. 05753

MARIO BALBI, Benedictine Mili School, 6502 Seawright Dr., Savannah, Ga. 31406

HUBERT BALCAEN, University of Manitoba, 500 Dysart Road, Winnepeg 19, Manitoba, Canada

MRS. JEANNIE BALL, Madeina School, Greenway, Va. 22067

MS. VIVIJA W. BALTINS, P. O. Box 1012, Ft. Wayne, Ind. 46801

PAUL P. BALTIS, 96-050 Farrington Hwy., Pearl City, Hawaii 96782

HUGO BANER, Lindenwood College, St. Charles, No. 63301

KENNETH S. BARGER, DLL, Columbia Community Unit 4, P. O. Box 1, Columbia, Ill. 62236

SHARON BARGER, Laboratory Supervisor, Woodstock High School, Woodstock, Ill. 60098

ELIZ M. BARICEVIC, Maryhurst College, Maryhurst, Oregon 97036

CHARLES J. BARR, DLL, St. John S. College, 5118 Seminary Road, Camarillo, Cai. 93010 
J. E. BARRETT, Hd. Lang. Dept., St. George S. School, W2929 Waikiki Road, Spokane, Wa. 99208

PEDRE BARRIE, DLL, Macon Junior College, Macon, Ga. 31206

DR. RICHARD BARRUTIA, Univ. De. Calif., Apartado Postal 6-884, Mexico 6 D. F., Mexico

REV. GILBERT BARTH, T. O. R., Head, Dept. of Mod. Lang., College of Steubenville, Steubenville, Ohio 43952

RALPH W. BASSETT, Portland Public Schools, 631 NE Clackamas Street, Portland, Ore. 97208

DR. MARGARET BATES, DLL, 5914 Carlton Lane, Bethesda, Maine 20016 TERESA BATTO, DLL, Loyola College, $4501 \mathrm{~N}$. Charles Street, Baltimore, Md. 21210

PHILIP R. BAUDIN, Coord., Highline Public Schools, P. O. Box 100, Seattle, Wash. 98166

HUGO BAUER, Lindenwood College, St. Charles, Mo. 63301

MLL. BAUMLIN, Milton Academy, 325 Randolph Avenue, Milton, Ma. 02186

DR. ERIC W. BAVER, Lang. Serv-Monash University, Clayton Vict., Australia

PAULINE BAXA, Dean, Dean Junior College, Franklin, Ma. 02038

MISS EVELYN N. BAXTER, Baldwin High School, Pittsburgh, Pa. 15216

MRS. ANTHONY BAYNARD, Maumee Valley Country Day School, 1715 S. Reynolds Road, Toledo, Ohio 43614

HENRI BEAUDOIN, Cegep De Rouyn-Noranda, P. O. Box 1500, Rouyn P, Quebec, Canada

MRS. JOHN BECK, 5832 S. Stoney Island, Chicago, Ill. 60637

MRS. JEAN BEECHER, Moravian College, Bethlehem, Pa. 18018

MISS YOLANDA C. BEH, Seamec Reg. Eng. Lang. Ctr., 104 Watten Estate, Singapore 11

DIANE BEIER, 709 Timpe Road, Fremont, Ohio 43420

KEITH R. BELL, Mid-American Nazarene College, 2030 College Way, Olathe, Kan. 66061

WILLIAM BEMERSDORFER, Ohio State University, Listening Center, Columbus, Ohio 43210

DR. MARIO A. BENITEZ, Chmn., Mod. Lang. Dept., Texas A \& I University, Kingsville, Tex. 78363

SR. GLORIA BENOIST, DLL, St. Mary S. Dominican College, 7214 St. Charles Avenue, New Orleans, La. 70118

PROFESSOR ANDREE BERGENS, Chmn., Dtpe. of French, Wilson College, Chambersburg, Pa. 17701

HENRI BERNIER, Walsh College, 2020 Easton Street, N. W., Canton, Ohio 44720

JAY BERNSTEIN, Nassau Community College, 79 Constable Lane, Levittown, N. Y. 11756

SR. M. BERNYNE, FSPA-APOF, Viterbo College, 815 South 9th Street, Lacrosse, Wis. 54601

MRS. DORIS BERTEAU, Horlick High School, 2119 Rapids Drive, Racine, Wis. 53404

PIERRE BIARNES, Macomb County Comm. College, 1450012 Mile Road, Warren, Mich. 48089

GILLES BIBEAU, Ch. Des. Lab/De Lang., Univ. De Montreal, C. P. 6128 (Faculte D Let), Montreal 3 Quebec, Canada 
REV. F. A. BIETER, Chmn. For. Languages, St. Thomas College, St. Paul, Minn. 55101

ELIZABETH M. BISHOFF, Randolph-Macon Womens College, R-MWC, Lynchbury, Va. 24503

PAUL R. BISSON, DLL, Greenfield Community College, 124 Federal Street, Greenfield, Ma. 01301

PROFESSOR CARL BLACK, Bard College, Annandale, N. Y. 12504

JOSEPH C. BLAIR, DLL, Lang. Lab A.S, University of Missouri, Columbia, Mo. 65201

CHARLES H. BLAYCHFORD, 476 Kekupua Street, Honolulu, Ha. 96821

R. V. BLOSJO, Alderson-Broaddus College, Phillippi, W. V. 26416

JAMES P. BLOUNT, DLL, College of William and Mary, Williamsburg, Va. 23185

BOARD OF EDUCATION, Freehold Regional High School District, 225 Schank Road, Freehold, N. J. 07728

PROFESSOR V. BOBETSKY, DLL, Polytechnic Institute of Brook, 333 Jay Street, Brooklyn, N. Y. 11201

HANS BODLANDER, University of Bridgeport, Dana Hall, Bridgeport, Ct. 06602

VIRA BODNAREK, DLL, Chicago City College, 3939 West 79th Street, Chicago, Ill. 60652

WILLIAM F. BOGART, APOG, Mansfield State College, 123 N. Williamson Road, Blossburg, Pa. 16912

J. CHARLES BOILES, Cegep St. Jean-Sur-Richelieu, Box 310, St. Jean PQ, Canada

MARCEL BOLDUC, Oblate College and Seminary, 149 Woodland Street, Natick, Ma. 01760

PROFESSOR R. BOLDUC, 251 Belmont Street, Quinsigamond Community College, Worcester, Ma. 01605

DR. G. DeBONE, La Grange College, LaGrange, Ga. 30240

WARREN C. BORN, Assistant Director MLA/ERIC, 62 Fifth Avenue, New York, N. Y. 10010

MARY BORRA, Supervisor, Warwich Veterans Memorial High School, Warwick, R. I. 02889

DEPARTMENT OF MODERN LANGUAGES, Boston University, 236 Bay State Road, Boston, Ma. 02215

JOHN C. BOUCHER, DLL, St. Amselm S. College, Manchester, N. H. 03102

ROGER BOUGARD, DLL, North Carolina College, 14061/2 Clarendon M., Durham, N. C. 27705

DR. H. A. BOURAOUI, 232 Founders Col. York U70, 4700 Keele Street Downsview, Ontario, Canada

FERNAND C. BOURASSA, Lord Beavercreek High School, 111 Grafton Drive SW, Calgary Alberta, Canada

DIRECTOR, LANGUAGE LAB, Bowdoin College, Brunswick, Me. 04011

LOUISE H. BOWEN, DLL, Dartmouth College, 201 Bartlett Hall, Hanover, N. H. 03755

MRS. STELLA M. BOY, Watertown High School, Watertown, Ma. 02172

COMMUNITY COLLEGE OF ALLEGHENY, Boyce Campus Lib/CTY, 595 Beatty Road, Monroesville, Pa. 15148

MRS. M. A. BOYDEN, Humanities Div., Royal College, Calgary Alta, Canada 
PATRICK C. BOYDEN, DLL, Kent State University, Kent, Ohio 44240

H. L. BRATNOBER, JR., SW Minnesota State College, Marshall, Minn. 56258

MRS. V. BRENTON, DLL, Vincennes University Junior College, Vincennes, Ind. 47591

KENNETH C. BRETT, University of Montana, Missoula, Mont. 59801

DIRECTOR, LANGUAGE LAB, Brevard College, Brevard, N. C. 28712

MRS. CABYRON B. BREYMAN, California Lutheran College, Thousand Oaks, California 91360

MRS. BECKY A. BRIGGS, Michigan State University, LL a-126 Wells Hall, E. Lansing, Mich. 48823

DR. ALFREDO BRIGOLA, DLL, University of Redlands, Redlands, California 92373

SR. M. CAROLYN BRINK, RSM, Our Lady of Cincinnati College, EdgecliffWalnut Hills, Cincinnati, Ohio 45206

MRS. F. C. BRITTAIN, W. Chicago Community High School, 326 Joliet Street, W. Chicago, Ill. 60185

ERIKA S. BROSSMAN, Albright College, Reading, Pa. 19604

DR. LUTHER F. BROSSMAN, Albright College, Reading, Pa. 19604

S. RAY BROST, Lycoming College, Williamsport, Pa. 17701

BROWN UNIVERSITY, Language Laboratory Library, Box E, Providence, R. I. 02912

C. P. BROWN, Western Kentucky University, Bowling Green, Ky. 42101

FLORENCE G. BROWN, Defense Lang. Ins. Sup. Com., 5312 Salisbury, El Paso, Tex. 79924

MARIE-LOUISE BRUGEYROUX, 1220161 Street, Edmonton 21 Alberta, Canada

WILBUR J. BRUNER, William Jewell College, Liberty, Mo. 64068

MRS. JUNE P. BRUST, UM Senior High School, Terwood Road, Willow Grove, $\mathrm{Pa}$.

MRS. M. A. BRYDON, Mount Royal College, Calgary, Alberta, Canada

SR. M. ALBAN BSHARAH, DLL, College of New Rochelle, New Rochelle, N. Y. 10801

BUCHHANDLUNG LEHMKUHL, 8000 Munchen 23, Leopoldstrasse 45, West Germany

DR. GEORGE C. BUCK, DLL, University of Washington, 118 Denny Hall, Seattle, Wa. 98105

BONNIE BUNDY, The Defiance College, L. C. Olds, Defiance, Ohio 43512 WILLIAM BURGESS, CCM Information Corp., 909 Third Avenue, New York, N. Y. 10022

MRS. CAROL A. BURLS, 1521 Urban Street, Baton Rouge, La. 70802

BURMA EDUCATION RESEARCH BUREAU, University P. O., Rangoon, Burma

YOLANDA BURR, Alfred University, RD No. 3, Wellsville, N. Y. 14895

THOMAS BUSHALLOW, Sup. FL, Shaker H. S., Latham, N. Y. 12110

MAGDELHAYNE BUTEAU, The St. Joseph Teachers College, 3465 Durocher Street, Montreal, Quebec, Canada

R. F. BYLINSKI, Institute de Ling App, 25 rue de Soleil, 67 Strasbourg, France 
JAMES C. CALLANAN, DLL, University of New Hampshire, Durham, N. H. 03824

CALVIN LIBRARY, 3207 Burton S. E., Grand Rapids, Mi. 49506

THOMAS F. CALVIN, DLL, Marshfield Public Schools, Marshfield, Ma. 02050

MRS. G. M. CAMPBELL, Senior High School, 160 Broad Street, Bloomfield, N. J. 07003

JOSEPH CANDIOTTI, United Nations Development Program, P. O. Box 24, Magadiscio Somalia E AF

PIERRE J. CAPRETZ, DLL, Yale University, 111 Grove Street, New Haven, Ct. 06510

CARLSBAD MUNICIPAL SCHOOL, Mid High School Library, 133 West Hagerman, Carlsbad, N. M. 88220

MAX CARMICHAEL, 607 West 77th Street, Indianopolis, Ind. 46260

JOSEPH A. CARRIER, 34 Birch St., Biddeford, Me. 04005

F. A. CARTIER, Defense Lang. Institute Eng., 7415 Buckskin Language School, San Antonio, Tex. 78227

RICHARD CARTWRIGHT, Mt. Hermon School, Mt. Hermon, Ma. 01354

CHRISTINE E. CERVIZZI, Northbridge High School, Linwood Avenue, Whitinsville, Ma. 01588

GERALD K. J. CHANG, 4457 Puu Panini Avenue, Honolulu, Ha. 96816

LOUIS J. CHATAGNIER, 4874 Cote Des Neiges, Apt. 1401, Montreal PQ, Canada

C. MAURICE CHERRY, DLL, Furman University, Greenville, S. C. 29613

RALPH CHERRY, DLL, Southeastern State College, Durant, Okla. 74701

LORA E. CHILDS, DLL, Wilson High School, 1151 SW Vermont Street, Portland, Ore. 97219

DR. ORLANDO CHOMAR, College of Mt. St. Joseph on the Ohio, 5701 Delhi Road,.Mt. St. Joseph, Ohio 45051

TOSHIKO CHOMEI, Ochanomizu University, Otsuka 2 Chome, Bunkyo-Ku, Tokyo, Japan

HENRY R. CHRISTMAN, DLL, Kutztown State College, 215 Wellington Avenue, Reading, $\mathrm{Pa} .19609$

A. E. CICERO, Allegheny Community College, 809 Ridge Avenue, Pittsburgh, $\mathrm{Pa}$. 15212

A. D. CICERO, 808 Ridge Avenue, Pittsburgh, Pa. 15212

NATHANIEL H. CIFFORD, Villa "Les Cigognes", Quartier Pierredon, 13 Eguilles, France

MISS JOAN E. CIRUTI, Mt. Holyoke College, So. Hadley, Ma. 01075

DR. A. W. CLAESGES, South Methodist University, Dallas, Tex. 75222

BEN T. CLARK, University of California, Merrill College UCSC, Santa Cruz, Ca. 95060

CLYDE L. CLARK, Associate Professor, Butler University, Indianapolis, Ind. 46208

DEPARTMENT OF ROMANCE LANGUAGES, Clark College, Worcester, Ma. 01610

EDWARD P. CLARK, Moorhead State College, Moorhead, Minn. 56560

THOMAS L. CLARK, English Department, University of Nevada, Las Vegas, Las Vegas, Nevada

CALVIN A. CLAUDEL, Box 622, Salisbury State Col., Salisbury, Md. 21801 


\section{Membership Directory}

MRS. J. V. COBB, 209 Black Bluff Rd., Rome, Ga. 30161

DR. FRANCESCA COLECCHIA, DLL, Duquesne Univ,, Pittsburgh, Pa. 15219

PAULINE COLL, Chmn., For. Lang., Averett Col., 135 Mulberry Rd., Danville, Va. 24541

RALPH S. COLLINS, Prof., Chmn. Dpt. FL, Maryville Coll., 1741 Linda Lane, Maryville, Tenn. 37801

RUTH W. COLOMBO, Lang. Dept., Natick H. S., 15 W. St., Natick, Ma. 01760

FOREIGN LANG LABORATORY, Colorado State University, Ft. Collins, Colorado 80521

COMMISSION SOLAIRE REG., Lalonde, Case Postale 3500, La Sarre Quebec, Canada

MRS. JEAN S. CONKEY, Supv. Lang. Lab, Wheaton Col., Norton, Ma. 02766 UNIVERSITY OF CONNECTICUT, Dir. Lang. Lab., Storrs, Ct. 06268

PAUL R. CONNORS, FL Coord. Randolph High, 194 H. St., So. Boston, Ma. 02127

DR. MARILYN J. CONWELL, DLL, Rosemont College, 251 W. Dekalb Pike, King of Prussia, $\mathrm{Pa}, 19406$

REV. DAVID P. Coon, A Hdmstr., Iolani School, 563 Kamoku St., Honolulu, Hi. 96814

C. T. COOPER, Asst. Prof. of Span., Lebanon Valley Col., Annville, Pa. 17003

JOHN CORPACI, Ch. Lang. Dept., Cheshire Aca., Box 306, Cheshire, Ct. 06410

BRO. H. MICHAEL CORRY, FSC-DLL, Christian Bro. Aca., Lincroft, N. J. 07738

ELADIO CORTES, DLL, Span. Dept. Chmn., Rutgers Univ., Camden, N. J. 08102

MRS. T. COSMAN, DLL, Barnard Col., 11 Lehman, 26 Claremont AVE/ HALL, New York, N. Y. 10027

RONALD KENT COSTA, DLL, Mass. St. Col., Framingham, Ma. 01701

NORMAN R. COTE, Head FLD, North Shore Comm. Col., 3 Essex St., Beverly, Ma. 01915

PAUL E. COTE, DLL, Avon High School, Avon, Ct. 06001

COTTEY COLLEGE, Blanch Skiff Memorial Library, Nevada, Miss. 64772

MADELEINE A. COURNOYER, Ch. FL, Provios East H. S., First Ave. and Madison, Maywood, Il. 60153

MARTIN J. COYNE, Tech. Asst. Lang. Lab, U. of Ma-Boston, 100 Arlington St., Boston, Ma. 02116

KARY NUTE CRAIGHILL, 4533 38th St., St. Petersburg, Fl. 33711

JOHIN F. CREAMER, DLL, Hudson H. S., Cottage St., Hudson, Ma. 01749

JOHN CRONIN, Lang. Coor., Maine-Endwell Central School, Endwell, N. Y. 13760

SR. M. ANSELM CROVER, DLL, Col. of the Holy Names, 3500 Mountain Blvd., Oakland, Ca. 94619

DR. DAVID CROWNER, DLL, Gettysburg Col., Gettysburg, Pa. 17325

J-P A. CRUVELLIER, Head Mod. Lang. Dept., Lower Canada Col., 4090 Royal Ave., Montreal 261, Canada

WILIIAM J. CUNEO, DLL, Holyoke Comm. Col., 170 Sargeant St., Holyoke, Ma. 01040

M. J. CUNNINGHAM JR., Ch. LD, New Mexico Junior Col., Lovington Hwy., Hobbs, N. M. 88240 
D. P. DACEY, KH 316, State U. Col. at Buffalo, 1300 Elmwood Ave., Buffalo, N. Y. 14222

BRO. ED DAILEY, Dept. Chmn., St. Edward H. S., 13500 Detroit Ave., Lakewood, Oh. 44107

RODNEY C. DALGO, DLL, Univ. of S. C., Lang. Lab., Columbus, S. C. 29208

MRS. RUTH V. DALY, 700 N. Main, Ada, Oh. 45810

EMMANUEL d'AMONVILLE, Chm., Foreign Lang. Dept., 745 Washington St., Braintree, Ma. 02185

MICHAEL DARRAS, Translation Bureau, Centennial Bldg., Frederiction, N. B., Canada

WALTER F. DAVISON, Old Dental Clinic, U. of Pittsburgh, Pittsburgh, Pa.

PHILIP STEPHEN DAY, Asst. Prof. Fr., Queen S. Univ., Kingston, Ontario, Canada

DAYTON BD. ED-SERV. Bldg., 4280 Northwestern, Dayton Ohio 45427, Att: Marguerite Turner

ALBERT EMANUEL LIBRARY, University of Dayton, Dayton, Oh. 45409

MISS DOLORES DECATOLI, LaSalle.Peru Twp. H. S., 541 Chartres St., LaSalle, Ill. 61301

JOHN DELANEY, Wolcott H. S., Wolcott, Ct. 06716

MRS. JOHN DELANOEYE, H. FLL, California State Col., 5151 State Col. Dr., Los Angeles, Ca. 90032

DE LA SALLE COLLEGE, Library, P. O. Box 3819, Manila, Philippines

MR. B. DEMAINE, 6971 Monkland Apt. 8, Montreal, 262, Canada

LARRY KENT DENNIS, D. Inst. Media, Concordia Col., Moorhead, Mn. 56560

SR. MARIE DE PAUL, Ch. Lang. Dept., Bishop Gallagher, 19360 Harper Ave., Harper Woods, Mi. 48236

REV. R. C. De PEAUX, Ch-Dept. of Mod. For. Lang., St. Norbert Col., W. DE PERE Wi. 54178

DEPT. OF ROMANCE LANG., University of N. C. at Greensboro, N. C. 27412 BENNIE T. DE SALVO, Hall High, 50 S. Main St., W. Hartford, Ct. 06051

DEPUTY LIBRARIAN, Centre for Info. on Lang. Teaching, State House High Holburn, London WC 1, England

LEGER DESROCHERS, 94 De L Acadie, Sept-Iles Quebec, Canada

DANIEL DETI, Chmn. Lang. Dept., Col. of the Desert, Palm Desert, Ca. 92260

EMILIO E DE TORRE, Queens Col. of the City of N. Y., Flushing, N. Y. 11367

MR. DIEGO DEVARGAS, FL Coor., Carlsbad Municipal School, 103 W. Hagerman St., Carlsbad, N. M. 88220

REV. F. J. DEVINE SJ, Chmn. Dept. Fr., St. Mary S. Univ., Halifax, N. S., Canada

JUSTINE M. DEVLIN, Head, MFL Dept., Lawrence H. S., Lawrence, Ma. 01841

MISS ELAINE DICICCO, Chmn. F. F. Dept., Concord-Carlisle High, Concord, Ma. 01742

WILLIAM C. DICKERMAN, DLL, Univ. of Houston, 3801 Cullen Blvd., Houston, Tx. 77004

ELMER DIETRICH, Hd. of Lang. Dept., High School, Miller, S. D. 57362 


\section{Membership Directory}

CARLO DIMAIO, DLL, Dept. Foreign Lang., Louisiana State U., Baton Rouge, La. 70803

MRS. MARGARET S. DINN, CNS I, Language Arts Dept., 1410 NE 2 Ave. Rm. 300, Miami, Fl. 33132

PROF. VITO DIVINCENZO, DLL, Villanova Univ., Spring and Hilldale Red, Malvern, Pa. 19355

DIV. OF INSTRUCTION, Midland Indpt. Sch. Dist., 702 North "N", Midland, Tx. 79701

FRANCE P. DIXON, DLL, McKendree Col., Lebanon, Il. 62254

HEADQUARTERS, DLIWC Aca Libr., Bldg. No. 302, Presidio Mntry, Ca. 93940

MICHAEL S. DODDY, Tch. Span., No Bergen H. S., 190 West 168th St., Bronx, N. Y. 10452

JAMES W. DODGE, University of Chicago Language Laboratory, 1126 East 59th Street, Chicago, Il. 60037

J. C. Doherty, DLL, St. Malachy S. H. S., Leinster St., St. John, N. B. Canada

ANDRE DOUESNARD, Aldt. Ed., Montreal Cath. Sch. Comm., 3737 E. Sherbrooke 406, Montreal, Canada

CHARLES DOWLING, DLL, Baldwin Wallace Col., Berea, Oh. 44017

MRS. B. DOWNING, West Tx. State Univ., Canyon, Tx. 79015

DR. O. DRAGONE, Dir., M. L. S., A. I. C., State St., Springfield, Ma. 01109

MR. EDWARD DRANEY, Chester Electronic Labs., 17 Navesink Dr., Monmouth Beach, N. J. 06412

DONALD L. DRUMMOND, Audio Coord., San Antonio Col., 1001 Howard St., San Antonio, Tx. 78212

SR. M. J. DUCHARME, Chmn. Mod. Lang., Rivier Col., Nashua, N. H. 03060

PIERRE R. DUCRETET, Asst. Dir., Prof. and Asst. LL Dir., Univ. College 213, U. of Toronto, Toronto 5, Ontario, Canada

M. ANDRE DUGAS, Dir., Department de linguistique, Universite du Quebec, Montreal, Que., Canada

DR. VYTAS DUKAS, Dir. For. LL, San Diego State Col., 5402 Col. Ave., San Diego, Ca. 92115

RICHARD DULKA, Dept. of For. Lang., Eastern IIl. Univ., Charleston, Ill. MR. V. du PREE, Lang. Lab. Supv., Mem. Univ. Newfoundland, St. John's NFL, Canada

AGNES DUNAWAY, Ch. FL, Riverside H. S., 1615 E. Locust St., Milwaukee, Wi. 53211

DR. H. B. DUNBAR, Ch. Div. of Lib. Arts, NYC Comm. Col., 300 Jay St., Brooklyn, N. Y. 11201

JOHN D. DURDEN, 4738 Everhart Dr., North Canton, Oh. 44720

BILL DURHAM, 201 Strode Tower, Clemson Univ., Clemson, S. C. 29631

F. A. DUVAL, Chmn. Clas. Mod. Lang., Cornell Col., 710 8th Ave. N., Mt. Vernon, Ia. 52314

$$
\text { - E - }
$$

R. M. ECONOMOS, Prof., Pace Col., 41 Park Row, New York, N. Y. 10038 MRS. JOHN EDIE, Ch. Fr. Dept., Northrop Collegiate Sch., 511 Kenwood Pkwy., Minneapolis, Min. 55403

CLAUDE EDLINE, DLL, Gilman School Inc., 5407 Roland Ave., Baltimore, Md. 21210 
EDUC. RESOURCES DEPT. ADMIN., Univ. of South Fl., 4202 Flowler Ave., Tampa, Fl. 33612

DAVID WAYNE EDWARDS, DLL, Emory Univ., Dept. of Romance Langs., Atlanta, Ga. 30322

W. EGGMANN, Chmn. Boys Div. L., Bis Reilly Diocesan H. S., F. Lewis and L. I. Expressway, Fresh Meadows, N. Y. 11365

RALPH A. EISENSTADT, DLL, West Chester State Col., For. Lang. Dep., West Chester, Pa. 19380

JUDY EISWERTH, 2828 Rowena Ave. No. 3, Los Angeles, Ca. 90039

EL CAMINO COLLEGE, Lang. Lab., 16007 S. Crenshaw Blvd., Via Torrance, Ga. 90506

MRS. JANE R. ELDON, DLL, No. Central Col., Naperville, Il. 60540

RICHARD H. ELDRIDGE, P. O. Box 46 W V W C, Buckhannon, W. V. 26201

MRS. LUCY ELIFSON, Waukesha H. S., 400 N. Grand Ave., Waukesha, Wi. 53186

RAY ELLIS, Prof., Hardin-Simmons Univ., Abilene, Tx. 79601

WILLIAM E. ELMENDORF, DLL, Western Wa. State Col., Bellingham, Wa. 98225

-ROGER J. ELMORE, AHMFL, Cumberland, Col., Box 121 Cumberland Col. St., Williamsburg, Ky. 40769

MAURICE G. ELTON, Southern Methodist Univ., Clements Hall, Dallas, Tx. 75222

EDMOND L. EMERY, Chmn. FL, Milford Academy, 150 Gulf St., Milford, Ct. 06460

SR. THERESE EMILIENNE, DLL, Annhurst Col., Woodstock, Ct. 06281

SR. MARY EMILY, Fr. Inst/DLL, 1330 Elmhurst Dr. NE, Cedar Rapids, Ia. 52402

ENGLISH LANGUAGE SCHOOL, DLIEL, LS Bldg. 7514, Lackland AF Base, Texas 78236

DR. FRANZ EPPERT, Assoc. Prof. Ger., Univ. of New Rrunswick, Fredericton N. B., Canada.

ALICE J. EPPINK, Lib., Center Applied Ling., 1717 Mass. Ave., NW, Washington, D. C. 20036

PROFESSOR CARL T. ERICKSON, P. O. Box 449, Shoreham, N. Y. 11786

MRS. EVELYN ERICKSON, Box 104, Mahopar, N. Y. 10541

LORENZO ESPINOSA, Lang. Lab. Supv., Univ. of California, Riverside, Ca. 92502

DR. ROBERT ESSA, Chrm. Fl. Dept., Sierra Col., 5000 Rocklin Rd., Rocklin, Ca. 95677

B. R. EWING JR., DLL, Washington and Lee Univ., Dupont 102, Lexington, Va. 24450

$$
-\mathbf{F}-
$$

MRS. H. FAILEY, 540 Jones Ave., Toronto 6, Ontario, Canada

MRS. HOPE FAIRLEY, DLL, 51 Terraulay St. (Bay Camp), George Brown Col., Toronto 102, Ontario, Canada

MAMIE FAIRLEY, Hd. FL, Magnolia H. S., Box 428, Moss Point, Ma. 39563

ILSE M. FANG, Prof., Suffolk Univ., 41 Temple St., Boston, Ma. 02114

BR. FARRELL CFX, Chrm. Mod. Lang., Ryken H. S., Leonardtown, Md. 20650 
JAMES C. FAULKNER, State Univ. College, 17 Cedar Ridge Rd., New Paltz, N. Y. 12561

MARIO R. FAYE, Chrm. For. Lang. Dept., Tacoma Comm. Col., Tacoma, Wa. 98465

LEO J. FECTEAU, Hd. For. Lang. Dept., Barrington Public Schools, Barrington H. S., Barrington, R. I. 02806

ROSA CASTRO FEINBERG, Robert E. Lee Jr. High, $3100 \mathrm{NW}$ 5th Ave., Miami, Fl. 33127

DR. WILLIAM M. FELSHER, Hd. Dept. For. Lang., Univ. of Evansville, Evansville, In. 47704

HIGH SCHOOL RESEARCH CTR., Felsooktatasi Pedagogiai, Kutatokozpont Budapest, VIII Rigo Utca 16 Hungary

DR. REST FENNER JR., Hd. Lang. Dept. 91 South Orange Ave., Livingston, N. J. 07039

LOU FERGUSON, Chrm. Fl. Dept., Glenbard East H. S., 1014 S. Main St., Lombard, Il. 60418

MARCIEN FERLAND, DLL, Univ. of Winnipeg, Winnipeg Man., Canada

DR. AUGUSTINE FERNANDEZ, Bishop Coll/A P R L, 3837 Simpson Stuart, Dallas, Tx. 75241

DR. JOSE C. FERNANDEZ, 6 Lakeside Court, Plattsburgh, N. Y. 12901

NINA FERSEN, DLL, The Karl E. Weston Lang. Ctr., Williams Col., Williamstown, Ma. 01267

A. V. FICARRA, Asst. Prof., Western Conn, State Col., 181 White St., Danbury, Ct. 06810

DOUGLAS A. FIELD, Dept. of For. Lang., Maui Comm. Col., Kahului Maui, Hi. 96732

BRUCE L. FINK, DLL, Pasadena City Col., 296 Ohio St., Pasadena, Ca. 91106

KARL T. FINK, Instr., Luther Col., 811 Iowa Ave., Decorah, Ia. 52101

B. J. FINN, DLL, Skill Center, 1485 Market St., San Francisco, Ca. 94103

MRS. G. FIRU, Univ of Ill., 305 Grant Hall, 1120 S. Morgan, Chicago, Ill. 60607

C. BRUCE FIRCH, DLL, Transylvania College, 300 N. Broadway, Lexington, Ky. 40508

FLEETWOOD FURN. CO., INC., Zeeland, Mi. 49464

R. FLEURANT, Pine Crest School, 1501 NE 62nd St., Ft. Lauderdale, Fl. 33308

ANTHONY FORD, DLL, Northeastern Univ., Boston, Ma. 02115

JOHN ELDON FORD, Instr. Fr., Northland Col., 213 and Street E, Ashland, Wi. 54806

FL DEPARTMENT, St. Cloud State College, St. Cloud, Min. 56301

DEBORAH FORTNER, DLL, Citrus H. S., Inverness, Fl. 32650

MLLE. DANIELE FOURNERET, Montreal Cath. Sch. Comm., Adult Education, 3737 E. Sherbrooke St., Montreal, Canada 406

JAMES F. FOURNET, DLL, Southeastern La. Col., 1004 S. Oak St., Hammond, La. 70401

ROBERT FOURNIER, Consult. FL, St. Dept. of Education, 17 Webster St., Suncook, N. H. 03275

BETTY FOWLER, Consult. FL, Dept. Ed., Stanislaus City SC, P. O. Box 1697, Modesto, Ca. 95354

DAVID FRANCISCO, Dir., Dial Access, Forest Pk. Col., 5600 Oakland, St. Louis, Mo. 63110 


\section{Membership Directory}

ANITA W. FRANKS, Span,, Thayer Academy, 745 Washington St., Braintree, Ma. 02185

GENE FRANKS, Dept. of For. Lang., No. Texas State Univ., Denton, Tx. 76203

ESTELLE C. FRAZIER, Fr. Tchr., Academy Heights H. S., P. O. Box 1210, Pinehurst, N. C. 28374

ERNEST A. FRECHETTE, Mod. Lang. Dept., Florida State Univ., Tallahassee, Fa. 32302

DOROTHY S. FREEMAN, Inst. Fr., Paine Col., 404 Sheffield Cir., Augusta, Ga. 30904

MR. ROBERT FREEMAN, West Bend H. S., 710 S. Main St., West Bend, Wis. 53095

DANIEL FREVAL, DLL, Dickinson Col., Dept. Mod. Lang., Carlisle, Pa. 17013

FRANK M. FRIEDMAN, DLL, Flint Comm. Jr. Col., 1401 E. Court St., Flint, Mi. 48503

HANS J. FRITSCH, P. O. Box 61, Whately, Ma. 01093

ARTHUR F. FROST, DLL, Princeton University, 304 E. Pyne St., Princeton, N. J. 08540

JOSEPH F. FUSCO, DLL, Dept. Eng. For. Stu., St. Michael S. Col., Winooski, Vt. 15404

$$
-\mathbf{G}-
$$

DOROTHY GABEL, Ch. F D, Resurrection High School, 7500 W. Talcott, Chicago, II. 60631

PROF. GILLES GAGNON, Royal Military Col. of Can., Massey Bldg., Kingston Ontario, Canada

H. F. GALLASCH JR., Dir. A-V Ctr., Davidson Com. Col., Box 1083, Lexington, N. C. 27292

THOMAS F. GALVIN, Dir. FL, Marshfield Pub. Sch., Marshfield, Ma. 02050

ALAN GARFINKEL, Tech., Dept. Educ. Assoc., Oklahoma State Univ., Stillwater, Ok. 74074

ERMAR E. GARINGER, DLL, Univ. of Kansas, Blake Annex, Lawrence, Ka. 66044

MR. GARY N. GARNER, Ch. FL, Dallas Baptist Col., P. O. Box 21206, Dallas, Tx. 75211

LOIS S. GAUDIN, Prof., Brooklyn Coll. of Cuny., Dept. Modern Lang., Brooklyn, N. Y. 11210

V. A. GAUDINO, Supv. LL, Lakehead Univ., Oliver Rd., Port Arthur Ontario, Canada

ROGER T. GAUTIER, DLL, Univ. of Saskatchewan, Saskatoon Sask., Canada

RICHARD A. GEIGER, Asst. Prof., Franklin and Marshall Col., Lancaster, Pa. 17604

MRS. ANNE H. GELINEAU, DLL, Harvard U. G-4 Boylston HL, Cambridge, Ma. 02138

GENO R. GEMMATO, Dir. Audio-Labs, Tufts Univ., Medford, Ma. 02155

N. GENDREAU, ML Dept., Rhode Island College, 600 MT Pleasant Ave., Providence, R. I. 02908

RAYMOND GENDRON, Hd. Lang. Dept., Seekonk Sr. H. S., Seekonk, Ma. 02771

MRS. M. G. GENNAULA, FL Chrm., Keystone Oaks H. S., 1000 Kelton Avc., Pittsburgh, $\mathrm{Pa}$. 15216 
UNIVERSITY OF GEORGIA, Dtpt. of German and Slavic Langs., Athens, Ga. 30601

DIRECTOR OF LANG., Div., German Center Boston, 170 Beacon St., Boston, Ma. 02116

GERMAN DEPT., F. J. Turner H. S., 1231 Inman Pkwy., Beloit, Wis. 53511

GEORGE GIANNETTI, Chrm. FL, Oak Park H. S., 13701 Oak Park Blvd., Oak Park, Mi. 48237

NATHANIEL H. GIFFORD, Hd. Mod. Lang. Dept., Dir. Lang. Lab., Brooks School, North Andover, Ma. 01845

SUSI GILBERT, DLL, Wofford Col., N. Church St., Spartanburg, S. C. 29301

S. ROSENDA GILL, DML, Lang. Lab. Coordinator, Regis College, Weston, Ma. 02193

MRS. SARA D. GILL, Mod. Lang. Dept., Scotland H. S., Laurinburg, N. C. 28352

ARTHUR J. GIONET, DLL, N. Texas State Univ., Denton, Tx. 76203

BRO. DENIS GIONET, DLL, Scolasticat Central, 7000 Marie-Victorin, Montreal, Canada 462

VIOLA GLENNIE, Instr., Alpena Comm. Col., 666 Johnson St., Alpena, Mi. 49707

MARCUS GOLDELL, Prof. of Span., 251 Belmont St., Worcester, Ma. 01605

E. M. GOLDSTEIN, Prof. Ed., Faculty of Educ/Grad Sch., University of Ottawa, Ottawa 2, Ontario, Canada

T. R. GOLDSWORTHY, DLL, Univ. of Wis., 866 Van Hise Hall, 1220 Linden Dr., Madison, Wi. 53706

ANGELITA FLORO GONZALES, California State Col., 27669 Loyola Ave., Hayward Ca. 94545

ALBERT A. GOODRICH, DLL, Joint School Dist. No. 1, Three Lakes, Wi. 54562

STEPHEN GOODYEAR, FLDH, Hull High School, 180 Main St., Hull, Ma. 02045

ALAN M. GORDON, DLL, Univ. of Toronto, Toronto, 5 Ontario, Canada

IAN B. GORDON, P. O. Box 507, Wingate, N. C. 28174

PETER B. GORE, DLL, Deerfield Aca., Deerfield, Ma. 01342

J. L. GOTLOBE, Comm. Col. of Phil. Lib., 34 S. 11th St., Philadelphia, Pa. 19107

SR. NOELLA GOULOT, Notre Dame Col., 2321 Elm St., Manchester, N. H. 03104

ALEX GRAMMATICOFF, Ch. FL D, Florissant Valley Comm., 3400 Pershall Rd. Col., St. Louis, Mo. 63135

GREEN VALE SCHOOL, Glen Head Long Island, New York, N. Y. 11545

JACK BYRON GREEN, DLL, Modesto Junior Col., Modesto, Ca. 95350

ESTELLE S. GREENE, DLL, Fairleigh Dickinson Univ., 207 Montross Ave., Rutherford, N. J. 07070

CLARA F. GREGORY, Spanish Consultant, Division of Program Development, 1202 Newning, Apt. 200, Austin, Tx. 78704

JOSEPH F. GREIPP, IDIT, 119 Sherwood Dr., Greenwood Pk., Blackwood, N. J. 08012

RONALD GRENNES, Dept. of Mod. Lang., VMI, Lexington, Va. 24450

MRS. AUDREY GREENWOOD, Chrm., Dept. of For. Lang., Idaho State Univ., Pocatello, Id. 83201 


\section{Membership Directory}

DR. MARCEL DE GREVE, Secretary, AIMAV, Avenue Georges Bergmann, 109, Bruxelles 5, Belgium

REV. J. J. GRIFFIN, Dir. A-V Lab., Allentown Col., Center Alley, Pa. 18034

DR. D. A. GRIFFITHS, Dept Fr., Univ. of Victoria, P. O. Box 1700, Victoria B. C., Canada

BILLIE G. GRIGG, Sup. L L, El Camino Col., 17204 Hass, Torrange, Ca. 90506

PAUL GRILLO, Hd. Fr. Dept., North Country School, Lake Placid, N. Y. 12946

FRANK GRITTNER, St. Supv/MFL, Wis. Dept. Pub Inst., 710 Woodward Dr., Madison, Wi. 53704

GLEN GROSJEAN, DLL, Univ. of Ca., B-40 Dwinelle Hall, Berkeley, Ca. 94720

ALLAN W. GRUNDSTROM, Asst. Prof. of Fr., Bucknell Univ., Lewisburg, $\mathrm{Pa} .17837$

PAUL G. GUENETTE, DLL, Town of Brookline, Town Hall, 333 Wash. St., Brookline, Ma. 02146

SR M. GEORGE GUERTIN, Mt. St. Mary Col., Hooksett, N. Y. 03106

DR. JAMES GUEST, Hd. Lang. Dept., Pensacola Jr. Col., 1000 College Blvd., Pensacola, Fl. 32504

EDWARD M. GUGGER, Hd. Dept. of FL, Medford H. S., Medford, Ma. 02155

DR. JEAN GUITTON, Chrm. ML Lab., Georgia College, Milledgeville, Ga. 31061

GUNARS VEVERIS, FL Dept., Thomas More College, Box 85, Covington, Ky. 41017

C. M. GUNDLACH, Supv, of L L, Wilmington Col., Box 990 Col. P. O., Wilmington, Oh. 45177

ROSALYN GURA, Supv. L L, Youngstown State Univ., 410 Wick Ave., Youngstown, Oh. 44503

KATHLEEN J. GUZEK, Fr. Tchr., Ontonagon Area H. S., 304 Pennsylvania Ave., Ontonagon, Mi. 49953

ERNESTO A. GUZMAN, Coord. Mod. FL, Tarrant Co. Jr. Col., South Campus, Ft. Worth, Tx. 76119

$$
-\mathrm{H}-
$$

MRS. DONALD F. HAGEN, DLL, Fairleigh Dickinson Univ., 285 Madison Ave., Madison, N. J. 07940

MORTON HAGUE, P. O. Box 10104, Oakland, Ca. 94610

ESTEHER HELENZ, Chrm., Columbia Union Col., Takom Park, Md. 20012 LEWIS HALL, Academic Dean, Lees-Mcrae Col., Banner Elk, N. C. 28604 WENDELL H. HALL, Asst. Prof. Span., Box 214 Univ. Station, Provo, Utah 84601

THE HALTON COUNTY BD. EDUC., P. O. Box 548, Oakville, Ontario, Canada

JOHN H. HAMMOND, Chrm., Texas Christian Univ., Dept. of Langs. Ft. Worth, TX. 76129

ERWIN M. HAMSON, DLL, Univ. of Michigan, 1401 Mason Hall, Ann Arbor, Mi. 48104

RAYMOND HAND, Instr. F L, Alexander City State Junior Col., Alexander City, Al. 35010 
DAVID A. HANSON, A P R, Ger. and Slavic Lang., Brandeis Univ., Waltham, Ma. 02154

JAMES HANSON, Chrm. Dept. of Mod. Lang., Adrian Col., Mahan Hall, Adrian, Mi. 49221

ERIC HAPBURN, 25 Calvin Ct., Old Bridge, N. J. 08857

JACOBINA HARDING, Hd. Fl Dept. and LL/Supv., Alamo Hts. H. S., 6900 Broadway, San Antonio, Tx. 78209

GLENN HAREWOOD, Humber Col. of AA \& T, Humber Col. Blvd., Texdale, Ont., Canada

T. J. HARRIMAN, VP Engr/Mkg., Conrac Corp., 330 Madison Avenue, New York, N. Y. 10017

BRO. EDWARD HARRISON, Fr. Sp. Inst., Walsh Col., 2020 Easton Rd., Canton, Oh. 44720

C. NORRIS HARRISON, Dir. Av. Services, Washington College, Chesterton, Md. 21620

FL DEPT., W. Hartford PS, 7 Whiting Lane, W. Hartford, Ct. 06119

G. CHRISTOPHER HARTLEY, Hd. Lang. Dept., The Peddie School, Hightstown, N. J. 08520

ALICE HARTMAN, Chrm. FL Dept., Joliet Twp. H. S. West, 401 N. Larking, Joliet IIl. 60435

MISS IRENE HASENCLEVER, DLL, Bennington Col., Bennington, Ct. 05201

V. HAUGHTON, Chrm. Fr. Dept., MacDuffie School, Springfield, Ma. 01107

MR. GUNTHER HAUK, Dept. Modern Languages, Allegheny College, Meadville, $\mathrm{Pa} .16335$

PAUL HAWKINS JR., A Ger. Chrm., W. Va. Univ., Parkersburg Ctr., 110 16th St., Vienna, W. Va. 26101

MISS YURIKO HAYASE, DLL, Winthrop Coll, Rock Hill, S. C. 29730

PAUL H. HAYES, D H F L, Framingham High School, A-Street, Framingham, Ma. 01701

CHARLOTTE HEDERVARY, Konth, Teacher, 3412 Main St., College Park, Ga. 30337

LILLIAN HEERMANS, DLL, Vassar Col., Poughkeepsie, N. Y. 12600

PROF. GEORGE W. HEFKE, Assoc. Prof. Fl., Kansas Wesleyan, Salina, Kansas 67401

DR. MAURICE M. HEIDINGER, Assoc. Prof. of Ger., Department of Foreign Language, Eastern Montana College, Billings, Montana 59101

ROBERT L. HENDRICKSON, 700 Broadway, Venice, Il. 62090

ADELE B. HERNANDEZ, Instr. of Span., 96 Falmouth St., Portland, Me. 04103

ROBERT F. HERON, Hd. FL Dept., Bellingham H. S., Bellingham, Ma. 02010

LAZARO M. HERRERA, DLL, The Westminster Schools, 1424 W. Pace Ferry Rd. NW, Atlanta, Ga. 30327

LINDA HEUBLEEN, DLL, Col. of St. Teresa, Winona, Mn. 55987

SHARON HEURERMAN, Supv. Lab., Woodstock High School, Woodstock, II. 60098

DR. J. HEWSON, Mem. Univ. of Newfoundland, St. John's NFL, Canada

HQTS-DLWIC ACADEMIC LIB., Bldg. No. 302, Presidio of Monterey, Monterey, Ca. 93940

CHARLES H. HICKMAN JR., 4025 8th St. So., St. Petersburg, Fl. 33705

DAVID B. HICKS, Hd. ML Dept., The Pingry School, North Ave., Hillside, N. J. 07207 
CENTRAL SERIALS SUBSCRIPTIONS, Hillman Library, University of Pittsburgh, Pittsburgh, Pa. 15213

MRS. MARGARETE HILTS, Loma Linda Univ., Riverside, Ca. 92505

MARGIE HINEKLEY, DLL, Inter American Univ., S. Tomas 134 URB Romani, Rio Piedras P. R. 00926

MRS. BERNICE G. HIRSCH, DLL, Samford Univ., 800 Lakeshore Dr., Birmingham, Al. 35209

ELTON HOCKING, Modern Lang. Educ., Purdue Univ., Coulter Hall, Lafayette, In. 47907

REUBEN W. HOLLAND, DLL, Univ. of Tennessee, Chattanooga, Tn. 37403 HOLLINS COL., DLL, Hollins Col., Va. 24020

JAY HOOT, Ger. Instr., Northland Col., Ashland, Wi. 54808

A. H. HOPKENS JR., Fr. and Eng., Middlefield Mem. Jr. High, Middlefield, Ct. 06455

A. H. HOPKINS JR., Tchr, Apt. G, 24 Jefferson St., Manchester, Ct. 06040

WILIIAM HORSTMAN, DLL, U. of Wis., Milw., Milwaukee, Wis. 53211

DEPT. of FL, Sam Houston State U., Huntsville, Tx. 77340

NORA C. HOWELL, DLL, Appalachian State Univ., Boone, N. C. 28607

RAYMOND HOWELL, 1403 3rd Ave. N. W., Seattle, Wa. 98177

ROBERT T. HOWLING, Chrm. Eng. Hum., New Haven Col., New Haven, Ct. 06505

DR. LOUISE J. HUBBARD, Chrm., D. C. Teachers Col., Div. of For. Lang., 11th and Harvard Sts. N. W., Washington, D. C. 20009

DONALD T. HUFFMAN, Prof., W. V. Univ., 781 Meadowbrook Rd., Morgantown, W. Va. 26505

EUGENE E. HUGHES, Chrm. Div. of Lang. Arts, Southwestern State Col., Weatherford, Ok. 73096

ALEX P. HULL JR., A P Lang., Tex. Tech. Col., DLL, P. O. Box 4285, Lubbock, TX. 79409

HARVEY J. HUMPHREY JR., DLL; Fordham University, Bronx, N. Y. 10458

MRS. MARY ELLEN HUNDLEY, Chrm. For. Lang. Dept., 405 36th Ave. N, Seattle, Wa. 98102

MR. WALTER HURLEY, Dept. Ger. CL Rus., Sacramento State Col., Sacramento, Ca. 95819

HERMAN HURTADO, Dir. Comm. Lab., FIA Atlantic University, Boca Ration, Fla. 33432

DR. JOSEPH C. HUTCHINSON, H. G. Defense Lang. Inst., 502 G. St. S. W., Washington, D. C. 20024

$$
-1 \text { - }
$$

PROF. D. A. IANNUZZI, Chrm. Mod. Lang. Dept., Niagara Univ., P. O. Box 164, Niagara Univ., N. Y. 14109

A. IBBOTSON, Dept. of French, Queen University, Kingston Ontario, Can. GEORGETTE L. IOUP, Stu., NYU, 402 W. 22nd St. N. 3F, New York, N. Y. 10011

DR. EVELYN IRVING, 1840 Azrock Drive, Knoxville, Tn. 37914

THE IRWIN LIBRARY, Butler University, Indianapolis, In. 46208

DR. MICHAEL ISSACHAROFF, Asst. Prof. Frnch-Dpt. Rom. Lang., U. of Chicago, Chicago, Il. 60637 


\section{Membership Directory}

\section{- J -}

DR. KENNETH JABLON, Prof. and Hd. of MFL, Frostburg State Col., Frostburg, Md. 21532

JAMES W. JACKSON, DLL, Wester Mich. Univ., Dept. of Mod. Lang., Kalamazoo, Mi. 49001

MARY E. JACKSON, DLL, Morgan State Col, Baltimore, Md. 21212

DR. W. RICHARD JACKSON, Coord. FLPalm Beech County, 3323 Belvedere Rd., W. Palm Beech, Fl. 33401

FERNAND JACQUES, Instr., Lakewood H. S., 13429 Emerson Ave., Lakewood, Oh. 44107

HANS JALIING, DLL Research Project, U. Stock-Strandbacken, Bromma, Sweden

MR. H. W. JAMIESON, 22 Thornlee Cr. N. W., Calgary 47, Alberta, Canda FRANCIS G. JARLETT, DLL, Central Ct. State Col., New Britain, Ct. 06050 CARL JERNER, 2600 Mission Bell Dr., San Pablo, Ca. 94806

ROBERT P. JOCHMANS, Chrm. ML Dept., Union College, Lincoln, Nb. 68506

RUTH M. JOHNGREN, Hd. FL Dept., Norwood Senior H. S., Norwood, Ma. 02062

BARBARA JOHNSON, Washington State University, Foreign Language Dept., Pullman, Wa. 99163

JANE P. JOHNSON, A Prof. Germ., Bakersville Col., 1801 Panorama Dr., Bakersfield, Ca. 93305

ROBERT JOHNSON, DLL, Claremont Men's Col., Claremont, Ca. 91711

MRS. VERONA JOHNSON, Lang. Lab. Aide, 4843 North 51 St., Milwaukee, Wi. 53218

A. JAMES JONES, Penn State University, S-320 Burrowes Bldg., University Park, Pa. 16802

R. J. JONES, The Col. of Ed., Univ. of Toronto 70, 371 Bloor St. W., Toronto 5, Ontario, Canada

ST. JOSEPH ACADEMY, 3200 Grand Ave., Des Moines, Ia. 50312

SAN JUAN, Jefferson State Jr. Col., 2601 Carson Road, Birmingham, Ala. 35215

SR. M. JUAN, Chrm. Fr. Dept., Regis Col., 235 Wellesley St., Weston, Ma. 02193

DENIS N. P. JUHEL, Dept. Romance Lang. UNB, Fredericton NB, Canada SR. JULIE, Hd. Fr. Dept., Maryvale-Trinity Col. Prep School, Valley Rd., Brooklandville, Md. 21022

$$
\text { - K - }
$$

DR. G. KADAR, DLL, Napa Col., Dept. of FL, Napa, Ca. 94558

EDMUNS J. KAMINSKI, Assoc. Prof. ML, Marietta College, Marietta, Oh. 45750

SHIRLEY J. KAUB, P. O. Box 2189, 545 W. Dayton St., Madison, Wi. 53703 YUSUKE KAWARABAYASHI, Dir. Univ. of Pacific, 145 Bernic Aye., Stockton, Ca. 95207

M. L. KAY, York Univ., Dir. Div. Ling. and Lang. Trng., Downsview, Ontario Canada

MISS P. KEENAN, 2682 17th Ave., San Francisco, Ca. 94116 
DENNIS KEHOE, DLL, Wisconsin State Univ., Whitewater, Wi. 53190

RITA KEHOE, Asst. DLL, Wisconsin State Univ., Route 2, Whitewater, Wi. 53190

DR. ANNA DALE KEK, Chrm. Lang. Dept., David and Elkins Col., Elkins, W. Va. 26241

MRS. CESI KELLINGER, DLL, Wilson Col,, Chambersburg, Pa. 17201

SR. BETH KELSO, DLL, St. Mary of Woods Col., St. Mary of Woods, In. 47876

PRISCILLA KELSO, DLL, Newton Jr. Col., Washington Park, Newtonville, Ma. 02160

JOSEPH KEMPRECOS, DLL, Rumson Fiar Haven Reg. Hi., Rumson, N. J. 07760

WILLIAM P. KENNEALY, DLL, Lexington H. S., 7 Hancock Ave., Lexington, Ma. 02173

DAPHENE KENNEDY, Supv. LL, Dept. of Mod. Lang., Freed-Hardeman Col., Henderson, Tn. 38240

SR. MARY KATHERINE KENT, Chrm. Mod. For. Lang. Dept., 103rd and Central Park Ave., Chicago, Il. 60655

LANGUAGE LABORATORIES, 312 Classroom Bldg., Univ. of Kentucky, Lexington, Ky. 40506

CHARLOTTE W. KERR, Supv. LL, Occidental Col., 1600 Campus Rd., Los Angeles, $\mathrm{Ca} .90041$

MRS. SYLVIA KIBART, Fl. Dept. Etsu., Commerce, Tx. 75428

R. D. KIEKEL, Asst. Prof., Dept. of Mod. Lang., Oregon State Univ., Corvallis, Or. 97331

DR. ARTHUR S. KIMMEL, For. Lang. Dept., Western Washington State Col., Bellingham, Wa. 98225

KINDERMAN (Canada) LTD, 3300 Cavendish Blvd., Suite 100, Montreal $261 \mathrm{PQ}$, Canada

ANNE KINSELL, HS Libr., W. Lafayette Comm., Leslie and Grant, Lafayette, In. 47901

PROF. THOMAS W. KIRKCONNELL, Chrm., Dept. of Mod. Lang., W. Va. Inst. of Tech., 114 4th Ave., Montgomery, W. Va. 25136

WALLACE L. KIZER, DLL, Baptist Col. at Charleston, P. O. Box 10087, Charleston, S. C. 29411

J. A. KLEINSASSER, Hd. Lang. Dept., Sioux Falls Col., Sioux Falls, S. D. 57101

JOHN K. KNUDSEN, DLL, Univ. of Pittsburgh, Dept. of General Linguistics, Pittsburgh, Pa. 15213

GEORGE E. KOEHLER, DLL, St. Procopius Col., Lisle, Il. 60532

SHIRLEY KOENEN, Instr. of Fr., 630 18th Ave., Lewiston, Id. 83501

DR. W. LAMARR KOPP, Asst. Dean, Penn State Univ., 138 Sparks Bldg., University Park, $\mathrm{Pa}$. 16802

PROF. TOM T. KOVARY, DLL, State Univ. of New York, Col. at Cortland, Cortland, N. Y. 13045

COMMANDANT G. KOZORIZ, Canadian Forces FLS, Dept. of Nat. Defense, Ottawa 4, Ontario, Canada

JACK B. KRAIL, D Audio L, Norfolk State Col., 2401 Corprew Ave., Norfolk, Va. 23502

DALE F. KRAMM, Instr. FL, Col. of St. Thomas, 1295 Blair Ave., St. Paul, Mont. 55104 


\section{Membership Directory}

MR. EDWARD L. KRUSE JR., Lect. in Ed., Canisius Col., Buffalo, N. Y. 14208 ROY L. KRYNITZ, DLL, For. Lang. Dept., New Mexico State Univ., University Park, N. M. 88001

PAUL F. KUCHAU, 215 McKay Bldg., Brigham Young Univ., Provo, Utah 84601

BETTY KUIEKA, Chrm. FL Dept., Kaimuki H. S., 2705 Kaimuki Ave., Honolulu, Hawaii 96816

\section{- L -}

QUENTIN LABELLE, Windsor Mountain School, Lenox, Ma. 01240

ARCH S. LACEFIELD, Sr. Instr., Col. Lib., Univ. of Ky., Henderson Comm. Col., Henderson, Ky. 42420

PAUL LACKAU, Brigham Young Univ., Lang. Lab. 215 McKay Bldg., Provo, Utah 84601

PH. G. LAFAURY, DLL, Univ. of St. Michael S. Col., 50 Sr. Joseph Sr. Fr. Dept., Toronto 5, Ontario, Canada

BRO. RICHARD C. LAFFERTY, LaSalle H. S., 8605 Cheltenham Ave., Philadelphia, $\mathrm{Pa} .19118$

L. A. LAFFORD, D A-V, St. Mary S Univ., Robie St., Halifax, N. S., Canada

SR. ELIZABETH LAFOREST, Pr. Fr., Mercy Col. of Detroit, 8200 W. Outer Dr., Detroit, Mi. 48219

RAYMOND J. LAGUEX, Act. Chrm. Dept. of Lang., Farmington State Col., Farmington, Me. 04938

DALE V. LALLY, DLL, Marquette Univ., Milwaukee, Wi. 53233

SR. REGINA MARIE LALONDE, OP, Siena Heights Col., Adrian, Mi. 49221

SR MONICA LANDRY, Notre Dame H. S., 910 N. Easton Ave., Crowley, La. 70526

DALE L. LANGE, Assoc. Prof., Univ. of Minnesota, 152-A Peik Hall, Minneapolis, Mn. 55455

JOHN B. LANGE, Assoc. Prof., St. Benedict S. Col., 526 Div., Atchison, Ks. 66002

TASSIE REE LANGLEY, A P E, Atlantic Christian Col., Dept. of Eng., Wilson, N. C. 27893

LANGUAGE H455, University of Minnesota, Duluth, Mn. 55812

LANG LAB DIRECTOR, Bergen Community College, Paramus, N. J. 07652

PROF. D. A. LANNUZZI, Chrm. Mod. Lang. Dept., Niagara Univ., P. O. Box 164, Niagara Univ., N. Y. 14109

DONALD LAPP, Prof. Span., Aurora Col., 347 Gladstone, Aurora, II. 60507

JAMES B. LARKIN, Assoc. Prof. Span., Coe Col., Cedar Rapids, Ia. 52402

ELINOR C. LARSON, DLL, Scotch Plains, Fanwood H. S., Westfield Rd., Scotch Plains, N. J. 07076

HELGA D. LASATER, Teacher's Aide, Montrey Penisula Col., 491 Hawthorne St. No. 8, Monterey, Ca. 93940

MARGARET LASHUA, DFL, Glastonbury Pub. Schools, Gideon Welles J. H. S,. Glastonbury, Ct. 06033

THOMAS A. LATHROP, A Prof. Mt. St. Mary's Col., Los Angeles, Ca. 90049 LANGUAGE LABORATORY, Laurentian Univ., Sudbury Ontario, Canada ADRIENNA LAVALLEE, DLL, Cumberland, R. I. 02864

JOSEPH P. LAWLOR, DLL, Lake Forest H. S., 1285 N. McKinley Rd., Lake Forest, Il. 60045

DONALD W. G. LAWSON, Dir., Paine Col., A.V Dept., Augusta, Ga. 30901 
AMADO M. LAY, Prof. Span., Gustavus Adolphus Col., Box 1413, St. Peter, Mn. 56082

S. PETER LEAHY, DLL, Wisconsin State Univ., Stevens Point, Wi. 54481

RAYMOND J. LEDINSKY JR., DLL, New Trier H. S. East, Winnetka, Il. 60093

BUCHHANDLUNG LEHMKUHL, 8000 Munchen 23, Leopoldstrasse, West Germany

CHARLES LEIGHTON, Chrm. Dept. Span. \& Classics - A P, Univ. of New Hampshire, Durham, N. H. 03824

J-C LEMYZE, DLL, McGill University, Montreal P. C., Canada

ARVELLA LENSING, DLL, St. Ambrose College, Davenport, Ia. 52803

ARTHUR A. LEONE, 2593 Shelly Dr., Indiana, Pa. 15701

MRS. B. LEVINSON, Supv. Lab., Brooklyn Col., City U., Bedford Ave. and Ave. H, Brooklyn, N. Y. 11210

DAVID W. P. LEWIS, Lakehead Univ., Port Arthur Ontario, Canada

MISS WYNELLE LEWIS, Hd. Span. Dept., DLL, Peace Col., Raleigh, N. C. 27602

LIBRARY RESOURCE CENTER, 201 E. Ning Mile Rd., Highland Springs, Va. 23075

\section{$-\mathrm{M}-$}

MARCEL LIDJI, Lecturer, Romance Lang. Lab Ccny., 133rd and Convent Ave., New York, N. Y. 10031

RICHARD L. LIGHT, DLL, Dept. Eng. For. Stu., St. Michael S. Col., Winooski, Vt. 15404

DAVID W. LIGHTCAP, Audio-Visual Dir., Susquehanna Univ., Selinsgroe, Pa. 17870

R. H. LINBERG, Chrm. Lang. Dept., Gov. Dummer Aca., Bayfield, Ma. 01922

FL DEPARTMENT, Lincoln H. S., 1433 S. 8th St., Manitowoc, Wi. 54220

LUIS LINDNER, DLL, Stephens College, Columbia, Mo. 65201

MRS. PIRKKO LINDQUIST, Alex von Humboldt Kielil, Turun YlipoistoTurku, Suomi, Finland

LINGUAPHONE INSTITUTE, 207-209 Regent St., London, England

DR. EDGAR A. LIST, Chrm. Dept. of For. Langs., Fresno State Col., Fresno, Ca. 93726

MRS. LINDA W. LITTLE, Dept. of Foreign Language, Northern Illinois University, DeKalb, Ill. 60115

S. JOSEPHINE LOMBARDO, Rosary Hill Col., 4380 Main St., Buffalo, N. Y. 14226

RUTH LOOMIS, FL Curr. Assoc., Madison Memorial H. S., 201 S. Gammon Rd., Madison, Wi. 53705

HEAD OF FRENCH DEPT., Lord Beaverbrook H. S., 9019 Fairmont Dr., SE, Calgary 30, Alberta, Canada

DR. A. G. LO RE, DLL, Univ. of North Carolina, 105 Dry Hall, Chapel Hill, N. C. 27514

MARIE LORRAINE LEE, Fr. Dept., Far Hills City Day School, Far Hills, N. J. 07931

ANNA MARIE LOTTMANN, Prof., Meramec Comm. Col., 959 S. Geyer Rd., Kirkwood, Mo. 63122

SR. ELAINE LOUBERT, Chrm., Col. of St. Scholastica, Duluth, Mn. 55811 
MRS. ROSABIANCA LOVERSO, Dept. Fr. and Ital., Sacramento State Col., Sacramento, Ca. 95819

ROBERT LOWENTHAL, DLL, Wesleyan Col., Rocky Mount, N. C. 27801

MICHAEL K. LUBIC, Asst. Prof., Miles Col., Birmingham, Al. 35203

BOYCE M. LUCAS, DLL, Whitehall H. S., Whitehall, Mi. 49461

PAUL F. LUCKAU, Asst. Prof., Brigham Young Univ., 215 McKay Bldg., Provo, Utah 84601

TTM LUIKART, DLL, Ashland Col., Ashland, Oh. 44805

ROLPH A. LUMPERT, University of Dubuque, German Department, Dubuque, Iowa

MRS. ANNA LYNCH, DLL, Trinity Col., Washington, D. C. 20910

MRS. B. G. LYTLE, Asst. Lab. T., Rd. 267 Warren St., Tully, N. Y. 13159

T. O. MACADOO, AP and DLL, Dept of For. Lang., Va. Polytechnic Inst., Blacksburg, Va. 24061

JOHN MACINKO, D FL Lab., Old Main Bldg., U. of Colorado, Boulder, Co. 80300

SR. RITA M. MACINNIS, CND., Xavier Col. Libr., Box 760, Sydney N. S., Canada

SR. MARIE MAGDALEN OP, DLL, Rosary Col., River Forest, Il. 60305

DR. ROBERT MAGILL, DLL, Towson State Col., Dept. Mod. Langs., Baltimore, Md. 21204

MICHAEL MAHLER, DLL, Univ. of Missouri, 8001 Natural Bridge, St. Louis, Mo. 63121

PIERCE MALI, ML Hd., Princeton Day School, The Great Rd., Princeton, N. J. 08540

G. THOMAS MANN, Instr. of Ger., Graceland Col., Lamoni, Ia. 50140

SR. MARGARET MANNING, DLL, Notre Dame Col. of SI, 300 Howard Ave., Staten Island, $\mathrm{N}$. $\mathrm{Y}$. 10301

JOHN C. MANOLIS, DLL, Mary Wasr. Col. of U. of Va., P. O. Box 1126, College Sta., Fredericksburg, Va. 22401

MRS. BARBARA MANTINI, N. Hennepin State Jr. College, 7411 85th Ave. N., Minneapolis, Minn. 55428

RACHEL MANWELL, L L Asst., Univ. of Illinois, 215 Lincoln Mall, Urbana, Il. 61801

ROGER E. MARESCHAL, 324 Nolin Apt. 4, Alma P. Q., Canada

DOROTHY MARKLE, Asst. Prof., Newark State Col., Morris Ave., Union, N. J. 07083

PROF. D. W. MARSHALL, Vancouver City Col., 912 West 12th Ave., Vancouver 9, BC, Canada

ROBERT T. MARSHALL, Chrm., Dept. of Lang. \& Ling., Mt. St. Mary S R Dl., Emmitsburg, Md. 21727

WILLIAM H. MARSHALL, Asst. Prof. of Lang., Univ. of Maine, Augusta, Me. 04330

RICHARD MARTI, 2369 Williams Apt. C. Columbus, Oh. 43202

BRO. JAMES MARTIN, CSC, Holy Cross H. S., 26-20 Francis-Lewis BL, Flushing, N. Y. 11358

WILLARD M. MARTIN, DLL, Penn State Univ., 6 Sparks Penn State Univ., University Park, Pa. 16802

CARLOS CORTES MARTINEZ, P. O. Box-1147, Bilbao-Vizcaya, Spain

JOHN MARTINEZ, Chrm. Span. Dept., New Trier Twp. H. S., 7 Happ Rd., Northfield, Il. 60093 


\section{Membership Directory}

DR. R. V. MARTINEZ, Chrm. Span. Dept., Franklin and Marshall Col., Lancaster, $\mathrm{Pa} .17601$

EDWARD MARXHEMMER, DLL, Univ. of Alberta, Edmonton, Alberta, Canada

ARNOLD H. MARZOLF, MDSU Mod. Lang. Dept., Fargo, N. D. 58102

GEORGE D. MASON, 22 Industrial Park Rd., Hingham Ma 02043

EDWARD C. MATRANGA, Supv. of For. Lang., Stratford Town Schools, Stratford, Ct. 06497

HELENE MATTHEWS, DLL, Adekphi Suffolk Col., 89 Collins Ave., Sayville, N. Y. 11782

DR. A. P. MATURE, Hd. Lang. Dept., Newberry Col., Newberry, S. C. 29108

WILLIAM C. MAXWELL, Hd. Lang. Dept., Tabor Aca., Box 372, Marion, Ma. 02738

MRS. RUTH ANN MAYS, Prof. of Fr., Pikeville Col., Pikeville, Ky. 41501

ALEX McANDREW, DLL, University of Sydney, Sydney, NSW, Australia

C. K. McCLATCHY, Library, 3066 Freeport Blvd., Sacramento, Ca. 95818

GEO. L. M. McCLINTOCK JR., 1825 McLaughlin Run Rd., Pittsburgh, Pa. 15241

DR. J. A. McCONOCHIE, DLL, N. Y. Univ., 435 East Bldg. NYU, New York, N. Y. 10003

MR. J. T. McCULLOUGH, Dept. Span. and Portuguese, Sacramento State Col., Sacramento, Ca. 95819

JERRY McCUNE, DLL, 120 Ballantine Hall, Indiana Univ., Bloomington, Ind. 47401

DR. KEITH A. McDUFFIE, Chrm. Dept. of FL, Univ. of Montana, Missoula, Mt. 59801

HELEN McGETTRICK, 115 St. Stephen St., Boxton, Ma. 02115

THOMAS McKEAN, HS Libr., 301 McKennan's Church Rd., Wilmington, Del. 19808

J. McKENNA, DLL, Col. of the Holy Cross, Worcester, Ma. 01610

MRS. McKENNA, Sec., Hyde School, Bath, Me. 04530

M. W. McKNIGHT, Assoc. Dir. Lang. Lab., Univ. of California, Irvine, Ca. 92664

JEAN McLANE, Asst. Prof., McPherson Col., McPherson, Ks. 67460

REV. J. McMANUS, U. of St. Thomas, 3812 Montrose Blvd., Houston, Tx. 77006

MRS. M. J. McNAMARA, Supv., Lang. Lab. Arts Bldg., Douglass Col., New Brunswick, N. J. 08903

JAMES J. MEADE JR., 35 McDevitt Rd., Randolph, Mass. 02368

MRS. CAROLL MEEKS, 1828 W. Pensacola St., Apt. B-14, Tallahassee, Fl. 32304

MR. WALTER MEIER, Seebacherstrasse, 8052 Zurich, Switzerland

UNIVERSITY OF MELBOURNE, Science Languages, 159 Barry St., Carlton Vic., Australia

PERIODICALS DEPARTMENT, Menash University Library, Clayton Vic. 3168, Australia

MERCED COLLEGE, 3600 "M" St., Merced, California 95340

ROBERT C. MERCHANT, Quality Electronic System, P. O. Box 246, Carmel Valley, Ca. 93924 


\section{Membership Directory}

ROY D. MERCHANT, P. O. Box 382, Athens, Ohio 45701

MARY JANE MESS, Chrm. FL Dept., Ottowa Twp. H. S., 211 E. Main St., Ottawa, Il. 61350

C. A. MESSNER, Assoc. Prof. Mod. Lang., Carleton College, Northfield, Mn. 55057

DR. JACQUES METFORD, DLL, University College UWO, London Ontario, Canada

F. B. METT, Inst., Res. at Bay, 1720 E. Capitol View HS, Milwaukee, Wi. 53211

DR. JOSEPH MICHEL, D. FL Ed. Cntr., U. of Texas, Austin, Tx. 78712

MICHALE MICKO, DLL, PMC Colleges, Chester, Pa. 19013

ARTHUR L. MICOZZI, Supv. FL, Board of Edu., Balti Co., 2401 Lampost Lane, Baltimore, Md. 21234

R. T. MIDDLETON, 3rd, DLL, Tougaloo College, Tougaloo, Ms. 39174

NORMAN MIKESELL, DLL, Indiana Univ., Ballantine Hall, Rm. 120, Bloomington, In. 47401

DR. E. KENNETH MILES, Professor in German, Univ. of Maine, 214 Little Hall, Orono, Me. 04473

EUGENE MULLER, Cncl. Mod. Lang., St. U. of NY at Buffalo, 250 Winspear Ave., Buffalo, N. Y. 14214

J. B. MILLER, Wittenberg Univ., Springfield, Oh. 45501

J. DALE MILLER, A P F and I, 360 McKay Bldg., Brigham Young Univ., Provo, Ut. 84601

MARTHA R. MILLER, Sec. to Dir., Harvard U. Mod. Lang. Ctr., Boylston Hall G3, Harvard, Cambridge, Ma. 02138

MYRON C. MILLER, Prof. Ger., Polk Junior College, 999 Ave. "H" NE, Winter Haven, Fl. 33880

ROBERT C. MILLER, Lang. Dept. Chrm., Thacher School, Ojai, Ca. 93023

DR. ROYCE W. MILLER, Chrm. ML, Gordon College, 255 Grapevine Rd., Wenham, Ma. 01984

SHELBY C. MILLER, 115 Dudley Rd., Cochituate, Ma. 01778

MRS. WILLIAM B. MILLMAN, Dir., Rider College, Trenton, N. J. 08602

DR. DOROTHY HURST MILLS, Dept. of For. Lang., Chapman College Lang. Lab., Orange, Ca. 92666

LANGUAGE DEPT. H 455, Univ. of Minn., Duluth, Mn. 55812

BERNARD MIREL, DLL, Purdue-Calumet, 6738 Magoun, Hammond, In. 46324

MARLIN M. MITTAC, 710 Garfield Ave., Aurora, Il. 60506

MARY E. MOEN, Instr., Anoka Ramsey State Jr. College, 7154 NE Riverview Terr., Minneapolis, Mn. 55432

PROF. GEORGE MOI, Camrose Lutheran College, Camrose Alberta, Canada

TAMARA S. MOISSIY, DLL, Curry College, Milton, Ma., 10 Mt. Pleasant St., Hyde Park, Ma. 02136

FRANCES MONGILLO, 179 Huntinghill Ave., Middleton, Ct. 96457

EDITH L. MONSON, Chrm. FL Dept., Georgetown College, Georgetown, Ky. 40324

COL. H. P. MONTAGUE, 196 Beach 128th St., Belle Harbor, N. Y. 11694

DEPT. DE LING. ET DE LANG. MOD., Univ. de Montreal, C P 6128, Montreal 3 P Q., Canada

DR. J. MICHAEL MOORE, Mesa College, San Diego, Ca. 92111 


\section{Membership Directory}

LIBRARY, SERIALS DEPT., Moorhead State College, Moorhead, Mn. 56560

SR. M. CATHERINE MORAN, OP, Edgewood Col. Sacred Heart, 855 Woodrow St., Madison, Wi. 53711

VAL GUY MOREAU, Portsmouth High, Education Lane, Portsmouth, R. I. 02871

MARGARET C. MORELY, DLL, Tchrs. Col-Columbia Univ., Box 66, 525 W. 20th, New York, N. Y. 10027

DR. ANTONIO MORENO, Span. Prof., Wash. \& Jefferson College, 752 Lockhart St., Washington, Pa. 15301

MRS. D. K. MORGAN, Dept. of Romance Langs., Univ. of Alberta, Edmonton 7 Alberta, Canada

MARGOT MORGAN, DLL, Sacred Heart Dominican College, 2401 E. Holcombe, Houston, Tx. 77021

ROBERT L. MORGENROTH, Hd. Dept. FL, 101 Watson Hall, Dekalb, Il. 60115

ROBERT R. MORRISON, C M L, Southern Missionary Col., P. O. Box 475, Collegedale, Tn. 37315

DORIS MORTON, Dir. Communications Lab., College of Emporia, Emporia, Ks. 66801

WORTH S. MOSER, Chmn. Dept. FL, Darlington School, Rome, Ga. 30161

CLIFF MOUNTAIN, D F L, Mesa College, 1120 North Ave., Grand Jct., Co. 81501

CHARLES V. MULHOLLAND, Univ. RI, Lang. Lab. Supv., Dept. of Langs., Kingston, R. I. 02881

EMILY MULLER, Librarian, Wilton Senior H. S., 363 Danbury Rd., Wilton, Ct. 06897

GERALDINA ORTIZ-MUNIZ, Dept. For. Lang., Eastern Illionis Univ., Charleston, Il. 61920

SR. EVELYN MURPHY, College of Great Falls, Great Falls, Mt. 59401

HAROLD T. MURPHY, A P Sp., Marshall Univ., P. O. Box 238, Huntington, W. Va. 25701

ANDREW H. MURRAY, Coord. Lang Lab., Suffolk Community College, P. O. Box V, Stony Brook, N. Y. 11790

$$
-\mathbf{N}-
$$

JAMES E. NABERS, DLL, Southern Ill. Univ., Dept. of For. Lang., Carbondale, Il. 62903

E. NACHREINEI, Assoc. Prof. of German, Andrews Univ., Berrien Springs, Mi. 49104

MRS. VALESKA S. NAJERS, ISLL, Northeastern Ill. St. College, 401 Berkley, Elmhurst, Il. 60126

LANGUAGE DIVISION, Napa College, 2277 Napa-Valleje Hwy., Napa, Ca. 94558

LEON ORLAN NARVAEZ, Tchng., U. of Mn., Asst., LL, 2030 Brewster St., Apt. 13, St. Paul, Mn. 55108

DONALD S. NASH, Roberts Wesleyan College, North Chili, N. Y. 14514

MODERN LANGUAGES, U. S. Naval Academy, Annapolis, Md. 21402

MR. CARROLL E. NAVES, DLL, Barry College, Box 495, Miami, Fla. 33161

ORRIN NEARHOOF, Iowa Dept. of Pub. Inst., 2745 Bennett Ave., Des Moines, Ia. 50310 
WILLIAM R. NEHER, Hd. Lang. Dept., Breck School, 4200 W. River Rd., Minneapolis, Mn. 55406

MARY H. NELSON, 1800 Clarke Dr., Dubuque, Ia. 52001

MRS. MONIQUE NEMMI, French Dept., Glendon Col., 2275 Bayview, Toronto 317 Ont., Canada

UNIV. OF NEVADA, Dept. of For. Lang and Lit., Reno, Nv. 89507

U. OF NEVADA LAS VEGAS, Library, Periodicals, Las Vegas, Nevada 89109

BERNARD NEWMAN, Tchr., Ch. Lang. Lab., Bayside High, 32-24 Corp. Kennedy St., Bayside, N. Y. 11361

JAMES L. V. NEWMAN, Simmons College, 300 The Fenway, Boston, Ma. 02115

RICHARD W. NEWMAN, Chmn. For. Lang. Dept., 625 Huntington Ave., Boston, Ma. 02125

LEONARD NEWMARK, Chmn., Dept. of Ling., Univ. of California, La Jolla, Ca. 92037

CHAS. E. NICHOLSON, CMFL, Kansas State Dept. of Ed., 120 E. 10th St., Topeka, Ka. 66612

RUTH A. NIXON, Chmn, FL, Wisconsin State Univ., La Crosse, Wi. 54601 THEODORA NORWICH, DLL, Western College for Women, Oxford, Oh. 45056

WITOLD NOVAK, DLL, Audio-Visual Ctr., Monmouth College, Monmouth, Il. 61462

J. STANLEY NUFFER, DLL, Portland State Univ., P. O. Box 751, Portland, Or. 97207

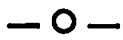

KARL W. OBRATH, Inst., DLL, Univ. of Cincinnati, 700 Riddle Rd. No. 202 Cincinnati, Oh. 45220

AMPARO B. OJEDA, LL Dept., Loyola Univ., N. Sheridan Rd., Chicago, Il. 60626

REV. AKRFED OKATIUST SH, Loyola H. S., 1901 Venice Blvd., Los Angeles, Ca. 90006

OKI.A. BAPTIST UNIV., Mod. Lang. Lab., Director, Shawnee, Ok. 74801

IRVING S. OLSEN, D Instr. Mats., Val Paraiso Univ., Mod. For. Lang. Rm. 147, Valparaiso, In. 46383

THE ONTARIO INSTITUTE, Dept. of Curriculum, 102 Bloor St. W., Toronto 5, Ont., Canada

BRUCE OTIS, City U. of New York, 158 E. 7th St., New York, N. Y. 10009

ROBERT OWENS, Chmn. Lang. Dept., Univ. of S. Alo., Mobile, Al. 36608

$$
-\mathrm{P} \text { - }
$$

TERRENCE PADEN, DLL, Upper Ia. College POB 583, Fayette, Ia. 52142 CLAUDE A. PAQUETTE, DLL, For. Lang. Dept., Univ. of Bridgeport, Bridgeport, Ct. 06602

F. A. PAQUETTE, Executive Sec., ACTFL, 62 Fifth Ave., New York, N. Y. 10011

ASSOC. PROF. B. E. PARIS, Faculty of Education, Sec. Dept. Regina Campus, Regina, Sask., Canada 
ARMANDO PARTINO, Defnse Lang. Inst., E C Br., U. S. Naval Sta., Navy Annex, Annacostia, Wa., D. C. 20390

DR. RAY PAST, Dept. of English, The Univ. of Texas, El Paso, Tx. 79999

LIBRARY, Parkland College, 2 Main St., Champaign, Il. 61820

GLORIA A. PATRON, DLL, La State Univ., Lake Front, New Orleans, La. 70122

E. PAUCKE, Dalton Hall, Bryn Mawr College, Bryn Mawr, Pa. 19010

CHRISTA E. PAUL, DLL, Pa. Col. of Pharm. and Sci., 43rd St. and Kingsessing Av., Philadelphia, Pa. 19147

PROF. RONALD S. PAZIK, Assoc. Prof., Univ. of Windsor, Windsor, Ontario, Canada

J. HUNTER PEAK, Hd. Prof. FLD, Auburn Univ., Mell Hall 205, Auburn, Al. 36830

ROSE D. PEARSON, Supv. Lang. Lab., Fairfield Univ., 1540 Unquowa Rd., Fairfield, Ct. 06430

PAUL D. PELOQUIN, Chicopee Comp. H. S., Rolf Ave., Chicopee Fallsy, Ma. 01020

GEORGE J. PENNEY H. S., 869 Forbes St., E. Hartford, Ct. 06108

PENSACOLA JR. COL., DLL, 100 Col. Blvd., Pensacola, Fl. 32504

RICHARD M. PENTA, Coord. of FL, 38 Normandy Rd., Lexington, Ma. 02173

PERIODICALS DEPT., College Library, Cal State Polytechnic, San Luis Obispo, Ca. 93401

PAUL W. PETERSON, Div. Dept. Mod. Lang., Gannon College, Erie, Pa. 16501

MR. D. L. PETHERBRIDGE, Assoc. Prof., Univ. of Lethbridge, Lethbridge, Alb., Canada

DR. MILTON I. PETRIE, Dir. Av/Tv Ctr., U. of Louisville, Louisville, Ky. 40208

REV. R. PETRIE, A-V Coord., St. Joseph Seminary, Callicoon, N. Y. 12723

DR. DANIEL J. PETRIZZI, Coord. FL, Eisenhower College, Seneca Falls, N. Y. 13148

MIRCEA PETRU, SC Asst., Kingsborough Comm. College, Box 700, Brooklyn, N. Y. 11235

JOHN A. PETTIT, Hd. ML Dept., Marietta College, Marietta, Oh. 45750

JANE P. PETTY, A Prof. Ger., Bakersfield Col. LL Coord., 1801 Panorama Dr., Bakersfield, Ca. 93305

J. E. PEYRAZAT, DLL, Roanoke College, Salem, Va. 24135

SR. M. PHYLLIS, Notre Dame Preparatory, 815 Hampton Lane, Baltimore, Md. 21204

MRS. ELSIE V. PICCO, Lab. Supv., Bennington College, Bennington, Vt. 05201

WILLIAM J. PILRANIS, DLL, R. I. Jr. College, 199 Promenade St., Providence, RI. 02908

MICHAEL S. PINCUS, Chmn. Span. and Classics, Univ. of New Hampshire, Durham, N. H. 03824

MISS GLADYS PIOLA, 194 Washington St. 3111, Hartford, Ct. 06106

MRS. I. L. PITT, D-Spvsr., Univ. of Virginia, 302 Cabell Hall, Charlottesville, Va. 22903

M. GUY PLASTRE, Faculte Des Lettres, Universite Laval, Quebec, Canada 


\section{Membership Directory}

RS. M. LELIA POND, DLL, Univ. of Dallas, Univ. of Dallas Station, Dallas, Tx. 75061

WILLIAM A. POPE, Dept. of Mod. Lang., Keuka College, Keuka Park, N. Y. 14478

JOHN PORTERA, DLL, San Fernando Valley State College, 1811 Nordhoff St., Northridge, Ca. 91324

B. J. PRADES, Sp. Instr., DLL, 444 Armstrong Hall, W. Va. Univ., Morgantown, W. Va. 26506

JUDITH PRATT, Chmn., Oswego H. S., West First St., Oswego, N. Y. 13126

DOMENIC R. PROCOPIO, Chmn., Dept. of For. Languages, Lowell State College, Lowell, Ma. 01854

PUBL. PROCUREMENT CTR., P. O. Box 9355, Arlington, Va. 22209

G. PRYTHERCH, Dept. Hd., 6567 Cypress St., Vancouver 14, B. C., Canada

SR. AGNES PURICELLI, CSJ Instr., Pontbonne College, 6800 Wydown Blvd., St. Louis, Mo. 63105

GUNTHER F. PUSCHENDORF, Sup. Lab., Univ. of California, Berk., 75 Menlo Ave., W. Lake Daly City, Ca. 95015

$$
\text { - Q - }
$$

MICHAEL P. QUERCIA, Belmont Public Schools, 644 Pleasant St., Belmont, Ma. 02178

MICHAEL T. QUEYJA, DLL, Univ. of Mississippi, University, Ms. 38677

T. QUINN, Grad. Stu., Ohio State Univ., 1384 Jones Tower, Columbus, Ohio 43210

J. RADTKE, Hd. Mod. Lang., Marquette H. S., 3401 W. Wi Ave., Milwaukee, Wi. 53208

HERMAN G. RADUNZ, Lang. Lab. Asst., Vancouver City College, I-3548 W. 4th Ave., Vancouver 8, B. C., Canada

MRS. JOSEPHINE RAFFERTY, 890B Gatehourse Dr., Decatur, Ga. 30032

LOUIS P. RAICHLE, Prof., Staten Island Comm. College, Ocean Terrace, Staten Island, N. Y. 10301

L. GRANT REESE, Chmn. Dept. of Mod. Lang., Memphis State Univ., Memphis, Tn. 38111

MR. WINSTON J. REESE, DLL, Univ. of Iowa, 125 Schaeffer Hall, Iowa City, Ia. 52240

DONA REEVES, Chmn. ML, Southwest Tx. State Univ., 1101 Robert E. Lee Rd., Austin, Tx. 78704

CONSTANCE L. REID, Eigenmann Cntr. Indiana Univ., Bloomington, In. 47405

MRS. FRANCES REID, Supvsr., Lang. Lab., Davidson College, Davidson, N. C. 28036

COL. HOWARD REINER, 37 Walnut Ave., Wheeling, W. Va. 26003

DR. GOTTFRIED REITINGER, Universtat Innsbruck, Fischnalerstrasse 4, 6020 Innsbruck, Austria

SR. ELIZABETH RHODE, Ch. LD, Alverno College, 3401 South 39 St., Milwaukee, Wi. 53215

CHARLES P. RICHARDSON, DLL, Ellis Hall, Ohio University, Athens, Ohio 45701 


\section{Membership Directory}

SR. JANET RICHARDSON, Chmn. FL Dept., Englewood Cliffs College, Englewood Cliffs, N. J. 07632

MR. J. RICHER, DLL, College Ste-Marie, 1180 Bieury, Montreal, 2 P. Q., Canada

ALBERT RIUSECH, A Prof. of Lang., Ouachita Baptist U., Box 739, Arkadelphia, Ar. 71923

PROF. RIVA, Haag Hall Rm. 304, Univ. of Missouri, Kansas City, Mo. 64110

MARGARETTE ROBBINS, Box 667, H-S, U. Sta., Abilene, Tx. 79601

DR. W. M. RIVERS, Dept. Mod. Lang., Monash Univ., Clayton Victoria 3168, Australia.

CLAUDETTE ROBINSON, 2135 Gilford, Montreal, Quebec, Canada

MARY LUNN RODGERS, Chmn. Dept. FL, Box 643 H-S Sta., Abilene, Tx. 79601

S. M. ROSELDA, Hd. Mod. Lang. Dept., Mater Dei H. S., 9th and Plum St., Breese, Il. 62230

SR. ROSENDA, D M L, Lang. Lab. Coord., Regis College, Weston, Ma. 02193

E. ROSHGOLD, 22 Mishmeret St., Afeka Tel-Aviv, Israel

CHARLES E. ROSS, Tchng. Ast., Univ. of Southern Calif., 10711 Rose Ave., Los Angeles, Ca. 90034

ROSS MEMORIAL LIBRARY, Cottey College, Nevada, Mo. 64772

MRS. ADA ROSSDALE, 435 East Bldg. 239 Greene St., New York, N. Y. 10003

PETER J, ROSTER JR., Asst. Prof. Carieton U., Colonel by Drive, Ottawa Ont., Canada

DR. PHILIP ROVNER, Univ. of Maryland, Dept. of Span. and Port., College Park, Md. 20742

PROF. J. ROY, Dept. Fr., Rm. A351 Arts Bldg., Lourention Univ., Ramsey Lake, Sudbury, Ontario, Canada

KATHLEEN KAY RUBIN, Regis College, W. 50th Ave. and Lowell Blvd., Denver, Co. 80221

PAULA RUFFIEUX, DLL, Rm. S 114 Ross Bldg., 4700 Keele St., York U., Downsview Ont., Canada

GORDON W. RUGGLES, Dir., Ashland College, College Ave., Ashland, Ohio 44805

MARCELLE RUMPF, Lecturer, Univ. of Lethbridge, Lethbridge Alberta, Canada

REV. MELVIN RUPPRECHT, DLL, St. Vincent College, Latrobe, Pa. 15650

NORMA RUSCH, DLL, Lakeland Union H. S., Minocqua, Wi. 54548

MRS. MARY RUTE, Tape Libr., Harvard U., Mod. Lang. Ctr., Boylston Hall, Harvard Yd., Cambridge, Ma. 02138

BETTY RYAN (1301), Scott Foresman Edit. Dpt., 1900 E. Lake Ave., Glenview, Il. 60025

MRS. CAROLE ANN RYAN, Asst. Prof. of Fr., Illinois College, Jacksonville, Il. 62650

ALLAN T. SABEAN, D A-V, Ctr., St. Marys Univ., Halifax, N. S., Canada CECIL SACHER, Chmn. FL, Niles Twp. H. S. N., 9800 Lawler, Skokie, Il. 60076

DR. LONNIE SADBERRY, Prof. of Span., Prairie View A \& M College, Prairie View, Tx. 77445 
SAMUEL A. SALAS, DLL, Cranbrook School, 520 Lone Pine Rd., Bloomfield Hills, Mi. 48013

MODERN LANGUAGE DEPARTMENT, Salem H. S., 29 Highland Ave,, Salem, Ma. 01970

DR. GRAYCE SALERNO, C D FL, Dept. Eng. \& For. Lang., Bergen Community College, Paramus, N. J. 07652

VICTOR SAMPON, Ch. FL, DLL, Wayland Academy, Beaver Dam, Wi. 53916

MRS. MARY SANCHEZ, Supv., For. Lang., P. O. Box 4688, Clearwater, Fl. 33518

SAN DIEGO STATE COLLEGE, FL Lab., Att: Vytas Kukas, 5402 College Ave., San Diego, Ca. 92115

ELEANOR SANDSTOM, DLL, School Dist. of Pa., Parkway and 21st St., Philadelphia, $\mathrm{Pa} .19103$

SAN JOAQUIN DELTA COL., 3301 Kensington Way, Stockton, Ca. 95204

BENIGNO F. SANTOMO, Sp. Dept., Richborourgh Jr. H. S., 197 Pine Dr., Crestview, Fl. 32536

BENIGNO F. SANTOMO, Esl. Teacher, P. 0. 1134, Redondo Bch., Ca. 90278

HELEN V. SAUNDERS, 1622 Franklin Ave., Charleston, W. Va. 25311

MRS. I. SAWYER, Dept. Mod. Lang., Simon Fraser Univ., Burnaby 2 B. C., Canada

J. O. SAWYER, DLL, Univ, of Ca., Berkeley, Ca. 94720

MARGARET SCARTH, Asst. Prof. in Span., Erindale Col.,-U. Toronto, Toronto 5, Ontario, Canada

THE LIBRARIAN, Secondary Tchrs. College College, Private Bag, Auckland, N. Zealand

JEAN PAUL SENECAL, 289 Gardenville, Longueuil, PQ, Canada

MISS RUTH SCHABACKER, 129 Kent Place Blvd., Summit, N. J. 07901

U. M. SCHAEFER, DLL, Connecticut College, Box 1524, New London, Ct. 06320

ANNE MARIE SCHAUBACHER, Chmn. Fr. Dept., Shipley School, Bryn Mawr, Pa. 19010

CARL W. SCHEFSKY, Inst. Med., Univ. of Portland, $5000 \mathrm{~N}$. Willamette Blvd., Portland, Or. 97203

EDWARD SCHEWE, 419 So. Taylor Ave., Oak Park, Il. 60302

VICTORIA SCHMITZ, Assoc. Dir., Schoonover Lab., Ohio Northern Univ., Ada, Ohio 45810

RONALD B. SCHMOLL, Asst. Prof. of Ger., Asheville, Biltmore College, Asheville, N. C. 28801

MRS. DIANNE SCHOBEL, 7801, Sagamore Dr., Cincinnati, Ohio 45236

FOREIGN LANGUAGE DEPT., Sch. International Tr., Kipling Road, Brattleboro, Vt. 05301

AURORA A. SCHOENFELD, Fl. Lab. Supervisor, U. of Southwestern La., Lafayette, La. 70506

EBERHARD SCHOLTIS, DLL, Plattsburgh State U. College, Plattsburgh, N. Y. 12901

GEORGE F. SCHULTZ, A Prof. of ML, Central State Univ., 109 N. Washington St., Columbia City, In. 46725

DENNIS M. SCHWANK, 4376 Declaration Drive, Indianapolis, In. 46227

D. SCHWENKER, IL MGR, Univ. of Guelph, 230 Edinburgh Rd. S., Geulph, Ontario, Canada

S. N. SCOTT, Instr. Ling., Kirkland College, Clinton, N. Y. 13323 
MRS. P. SCULLY, R P 1285 Victoria St., Toronto Ontario, Canada RUSSEL H. SEARCH, D A-V Materials, Bucknell Univ., Lewisburg, Pa. 17837 MOD. Lang. Dept., Seattle Comm. College Sys., Washington Community College Dist. VI, 1718 Broadway, Seattle, Wa. 98122

ROBERT P. SERAFINO, Supv. of F L, New Haven Pub. Sch., P. O. Box 3816, New Haven, Ct. 06525

CARLOS SERRANO, Stu., 75 Alexandra St., Waterloo Ontario, Canada

LIBRARY SERVICES, St. Dept. of Educ., 4500 Maccorkle S. E., Charleston, West Virginia

MRS. EILEEN SEXTON, Bronx Comm. College, 120 E. 184 St., Bronx, N. Y. 10468

MRS. MARY SHAPIRO, State College, Bridgewater, Ma. 02324

P. B. SHARPE, Dean Jr. College, 132 Main St., Franklin, Ma. 02038

JULIE SHEARER, Asst. Prof., Central Wesleyan College, Edgewood Arms No. 4, Clemson, S. C. 29631

PROF. JOSEPH H. SHEEHAN, Chmn. Faculty Comm. on Lang. Lab., Georgetown Univ., Washington, D. C. 20007

WALTER A. SHEETS, F L Supv., Bartholomew Cons. Sch. Corp., 1400 25th St., Columbus, In. 47201

ROBERT SHERBURNE, Cazenovia Central Sch., 2 Evergreen Ln., Cazenovia, N. Y. 13035

DAVID A. SHERRARD, Chief AV Br DLIEC, U. S. Naval Sta. - Anacostia An, Washington, D. C. 20390

MISS RENEE SHERROW, 315 E. 70th St., Apt. 2A, New York N. Y. 10021

MARION D. SHIELDS, MFL Dept. Hd., Dunedin H. S., Dunedin, Fl. 33528

MARY M. SHIREY, 2419 Longview Ave. S. W., Roanoke, Va. 24014

MRS. NAJLA SHOWKER, 102 Terrace Ct., Apt. 8 B, Johnson City, Tn. 37601

E. H. SHREFFLER, DLL, Austin College, Box 1239, Sherman, Tx. 75091

MR. DALE L. SIEVERS, 33 Murfreesboro Rd., Nashville, Tn. 37210

DOUGLAS J. SIMMONS, Asst. Pr., Dept. of For. Lang., Arizona State Univ., Temple, Az. 85281

HOWARD L. SIMMONS, DLL, Forest Park Comm. College, 38 Plaza South, Apt. 705, St. Louis, Mo. 63103

S. J. JOSEPH SIMMONS, Hd. Georgetown Prep. Sch. ML, 1225 Otis St. NE, Washington, D. C. 20017

JOHN SIREVAAG, Hd. Ger. Dept., Augustana College, Rock Island, Il. 61212

PETER SKANSE, DLL, Wheaton College, 425 E. Oak Ave., Wheaton, Il. 60187

RUTH SKWAREK, Instr., Muskegon City Comm. College, 15088 W. Leonard Rd., Spring Lake, Md. 49456

ALFRED N. SMITH, Asst. Prf., Dept. of Languages, Utah State Univ., Logan, Utah 84321

FRANCIS T. SMITH, DLL, Seton Hall U., So. Orange, N. J. 07079

DR. PHILIP D. SMITH, P C, West Chester State College, High and Rosedale Ave., W. Chester, Pa. 19380

WM. FLINT SMITH, DLL, Purdue Univ., Dept. of Mod. Lang., Lafayette, In. 47907

WILLIAM J. SMITHER, Prof. Sp., Tulane Univ., DLL, New Orleans, La. 70118 
ALEX SOKALSKI, Univ. of Bridgeport, Dept. of FL, Bridgeport, Ct. 06602 HENRY N. SOKOLOW, DLL, Santa Monica City College, Santa Monica, Ca. 90401

SERIALS DEPARTMENT, Somerset County College, P. O. Box 3300, Somerville, N. J. 08876

RECEIVING DEPARTMENT, Sonoma State College, 1801 E. Cotati Ave., Rohnert Park, Ca. 94928

DENNIS SOULE, Instr., Bethany Lutheran Jr. College, Mankato, Mn. 56001 LIBRARY, So. Brunswick H. S., Major Road, Monmouth Jct., N. J. SO. ILL. UNIVERSITY LIBR., Periodical Rding Clerk, Carbondale, Ill. 62901 FREDERICK A. SPEAR, Assoc. Prof. of Fr., Skidmore College, Saratoga Spgs., N. Y. 12866

PATRICK SPENCE, Asst. Dir., Baptist College, P. O. Box 10087, Charleston, S. C. 29411

ANTHONY SPETRINO, Chmn. FLD, Bassick H. S., 1181 Fairfield Ave., Bridgeport, Ct. 06605

O. R. SPINKS, Dir., Suny At Albany, Humanities B-16, Albany, N. Y. 12203 PROF. EDWARD M. STACK, 3925 Arrow Dr., Raleigh, N. C. 27609

MR. B. W. STADT, Span. Prof., Rollins College, Box 119, Winter Park, Fl. 32789

STANFORD UNIV. LIBRARIES, Order Department, Stanford, Ca. 94305

FL DEPARTMENT, St. Cloud State College, St. Cloud, Mn. 56301

ADRIAN L. STEINBERG, D FLL, Temple Univ., 3rd Floor, College Hall, Philadelphia, $\mathrm{Pa} .19122$

RHODA STERN, 305 E. 24th St. 18B, New York, N. Y. 10010

STETSON UNIVERSEITY, Lang. Dept., Box 1315 Univ. Sta., Deland, Fl. 32720

EDITH STEVENS, Methods Ins., Moorhead State College, 1211 4th Ave. S., Moorhead, Mn. 56560

ADLAI E. STEVENSON H. S., Attn Library, Prairie View, Il. 60069

HARRY E. STEWART, 600 South College, Tulsa, Ok. 74104

MR. JAMES J. SULLIVAN, 4914 Main St., Lisle, Il. $6 \$ 532$

JAMES J. SULLIVAN, Pres., James J. Sullivan Corp., 1517 Ogden Ave., Downers Grove, Il. 60515

SUMMIT LAB DIRECTORS, Board of Education, 97 Maple St., Summit, N. J., 07901

SUPERINTENDENT OF SCHOOLS, P. O. Box 1167, Darien, Ct. 06820

LOUIS M. SUTTON, Prof. of Fr., Baylor Univ., Waco, Tx. 76703

EDWARD S. SWAIN, Mod. Lang. Lab. Tech. Dir., Marquette Univ., Milwaukee, Wi. 53233

MR. SEVERIN A. SWANSON, RR1, Box 135, Omro, Wi. 54963

DR. DOUGLAS SWETT, Chmn. Dept. FL, Princopia College, Elsah, Il. 62028

MRS. MARTHA H. SWIFT, Morgan Park Academy, 2153 West 111th St., Chicago, Il. 60643 


\section{Membership Directory}

GILBERT TAGGART, Prof., Sir Geo. Williams Univ., 1435 Drummend St., Montreal 107 P. Q., Canada

MRS. J. M. TANNER, Dir. Supv., Univ. of Virginia, 302 Cabell Hall, Charlottesville, Va. 22903

DR. A. F. TARAS, D A-V Ctr., Ithaca College, Ithaca, N. Y. 14850

WALTER C. TAYLOR, Dept. of Span., Upsala College, E. Orange, N. J. 07019

TEACHERS COLLEGE LIBRARY, 525 W. 120th St., New York, N. Y. 10027

LANGUAGE LABORATORY, Tennessee A \& I State Univ., Nashville, Tn. 37203

THE U. OF TN. AT MARTIN, Rm. 427 Humanities Bldg., Martin, Tn. 38237

UNIVERSITY OF TEXAS, Language Laboratories, Batts Fall 227, Austin, TX. 78712

ACQUISITIONS LIBR., N. Texas State Univ., Box 5188, N T Sta., Denton, Tx. 76201

J. THATCHER, Asst. Hd/Mod. LD, Burlington Central H. S., Box 5042, Burlington, Ontario, Canada

SR. MARIE THERESE, Chrm. FL Dept., Good Counsel College, 52 N. Broadway, White Plains, N. Y. 10603

MARCELLA THERRIEN, DLL, 117 Margern Rd., Welland, Ontario, Canada

MR. JEAN R. THEUMA, D FL, Univ. of Hawaii, 2528 The Mall, Rm. 303, Honolulu, Hawaii 96822

CLAUDE THIBAULT, Ecole Normale N-D-De Foy, Cap-Rouge P. Q., Canada

DONALDO J. THOMANN, Pacific Union College, Angwin, Ca. 94508

FREDERICK M. THOMAS, Carlson H. S., 30550 W. Jefferson Ave., Gibralter, Mi. 48173

HALEY F. THOMAS, Instr., P. O. Box 403, Univ. of Richmond, Richmond, Va. 23173

GAIL THOMPSON, LL, North Central College, Rall Hall, Naperville, Il. 60540

E. F. THORNE, Assoc. Prof., West Liberty State College, West Liberty, W. Va. 26074

DONALD E. THORNHILL, DLL, Westport Bd. Education, Westport, Ct. 06880

JONATHAN TIBBETS, Acting DLL, Univ. of Cal. At Irvine, Ca. 92664

DR. M. J. TOCONITA, Prof. and Dir. L L, St. Joseph S. College, Philadelphia, Pa. 19131

THOMAS C. TODD, Dir., MATL, School For Intal. Training, Kipling Rd., Brattleboro, Vt. 05301

DOROTHY M. TOIRAC, Chmn. Lang. Dept., Grace College, Winono Lake, In. 46590

JON M. TOLMAN, Asst. Prof., Indiana Univ., Dept. Span. \& Port., Bloomington, In. 47401

DR. B. TOMKIN, St. Bonaventure Univ., St. Bonaventure, N. Y. 14778

A. J. TORRIELLI, 820 North Michigan Ave., Chicago, Il. 60611

PATRICIA N. TORNATORE, Pemus Valley Area H. S., Spring Mills, Pa. 16875

AUDUN TOVEN, Asst. Prof., Pacific Lutheran Univ., Taccma, Wash. 98447 ROBERT J. TRACY, DLL, Ridgewood H. S., Ridgewood, N. J. 07450 
ROGER R. TRAHAN, DLL, Assumption College, 500 Salisbury St., Worcester, Ma. 01609

DR. MANFRED TRIESCH, Dir., German Center, Boston, 170 Beacon St., Boston, Ma. 02138

MRS. GERTRUDE M. TRIPP, DLL, Elmhurst College, 190 Prospect Ave., Elmhurst, Il. 60126

MARGUERITE J. TUMER, Consint., Dayton Bd. of Education, 354 Bungalow Rd., Dayton, Ohio 45417

CAROLYN M. Ummel, Valley View H. S., RR 2, Box 3370, Germantown, Ohio 45327

UNIVERSITY OF MELBOURNE, Science Languages, 159 Barry Street, Carlton

UNIV. COL. OF RHODESIA, Lang. Lab., P. O. Box MP 167, Mount Pleasant Salisbury, Rhodesia

U. OF WESTERN ONTARIO, General Library, Lawson Memorial Bldg., London, Ontario

UNIVERSITY COLLEGE OF RHODESIA, Language Laboratory, P. 0. Box MP167, Mt. Pleasant Salisbury, Rhodesia

$$
-\mathrm{V} \text { - }
$$

MR. ALFIE A. VACANTI, 1506 Iverson St. No. 102, Oxon Hill Md. 20021

DR. LUIS J. VALVERGE, Ch. FLD, Boise State College, Box 24, Boise, Id. 83701

SR. EVANGELA VANACORE OP/Dir., Ohio Dominican College, 1216 Sunbury Dr., Columbus, Ohio 43219

MARY D. VANDESTEEG, DLL, Indiana Hill Jr.-Sr. H. S., 7370 Shawnee Rd., Apt. 3, Cincinnati, Ohio 45243

PAUL D. VANG, DLL, Thiel College, Greenville, Pa. 16125

SR. KATHRYN VAN HULST, Span. Teacher, Columbus H. S., Marshfield, Wi. 54449

JOHN W. VANKERK, DLL, Indiana State Univ., 15321/2 N. 7th St., Terre Haute, In. 45705

E. C. VAN MERKENSTEIJM, AD/Lang. Lab., 320 Logan Hall, Univ. of Pa., Philadelphia, $\mathrm{Pa} .19104$

GLENN J. VAN TREESE, DLL, Sweet Briar College, P. O. Box 18, Sweet Briar, Va. 24595

GRACE R. VAN WALK, Grants Pass High School, 522 N. E. Olive, Grants Pass, Or. 97526

DIRK B. VAN ZAANAN, DLL, Rutgers State Univ., 175 Univ. Av-Conklin Hall, Newark, N. J. 07102

PROF. HUGH E. VELTMAN, Grand Valley State Col., 615 Monroe Blvd., S. Haven, Mi. 49090

DAVID H. VERHALEN, Chmn. ML, Notre Dame H. S., 7655 Dempster St., Niles, Il. 60648

BRO. FRANCIS VESEL, Teacher, St. Michael S. H. S., Sante Fe, N. M. 87501 MICHELE VICTOR, DLL, Jamestown Comm. Col., 525 Falconer St., Jamestown, N. Y. 14701 
NEDDY A. VIGIL, DLL, Univ. of New Mexico, Dept of Mod. and Clas. Lang., Albuquerque, N. M. 87106

FL DEPT, Box 9, Virginia State College, Petersburg, Va. 23803

M. I. VOGT, DLL, Univ. of Idaho, ad 322 Univ. of Id., Moscow, Id. 83843

$$
-\mathrm{W}-
$$

SR. MARIE ANDRE WALSH IHM - DLL, St. Edward S. Univ., 300. S. Congress, Austin, Tx. 78704

LANGUAGE DEPARTMENT, Wardlaw County Day School, 1030 Central Ave., Plainfield, N. J. 07060

DR. VIRGIL A. WARREN, 323 Corprew St., Fayette, Mo. 65248

ELEANOR A. WASHINGTON, DLL, Nettie Lee Roth H. S., 727 Oak Leaf Dr., Dayton, Ohio 45408

FL DEPT. LANG. LAB., Washington University, St. Louis, Mo. 63130

JOHN A. WATSON, DFLL, Virginia Union University, Richmond, Va. 23220 DR. HARRY C. WEBB, D A-V, College of St. Thomas, St. Paul, Mn. 55101

DONALD B. WEBBER, Asst. Prof. Lang. Lab., The Univ. of the South, Sewanee, Tn. 37375

ROBERT WEE, Teacher, Ephrata H. S., 803 Oak Blvd., Ephrata, Pa. 17522

DR. ARNOLD L. WEINKAUF, Asst. Prof. of Language, Michigan Technological Univ., Houghton, Mi. 49931

THE WESTERN COLLEGE, Mod. Lang. Dept., Oxford, Ohio 45056

U. OF WESTERN ONTARIO, General Library, Lawson Memorial Bldg., London Ontario, Canada

ADELAIDE WHAN, DLL, Univ. of Southern California, Dept. of Religion, Los Angeles, Ca. 90007

D. W. WHEELEY, D A-V Educ., Brookline H. S., Brookline, Ma. 02147

DR. W. WHEETLEY, Dr. AV Education, 194 Boylston St., Brookline, Ma. 02146

RICHARD J. WHITE, DLL, Rich Central High Sch., Olympia Fields, Il. 60461

SIDNEY P. WHITE, Dept. Chrm., Morgan H. S., Route 81, Clinton, Ct. 06413

WHITEHALL-YEARLING HIGH SCHOOL LIBRARY, 675 S. Yearling Road, Whitehall, Ohio 43213

G. L. WHITINGTON, DLL, So. Texas Jr. College, Houston, Tx. 77002

ALLEN P. WHITMORE, Ch. Div. of Lang. and Lit., N. E. Missouri State College, Kirksville, Mo. 63501

KARL WIDMAIER, Modern Lang. Instr., Atlantic Union College, S. Lancaster, Ma. 01561

PETER WIESE, DLL, Southern Ct. State College, 501 Crescent St., New Haven, Ct. 06515

RAYMOND A. WILEY, Assoc. Prof., Lemoyne College, Syracuse, N. Y. 13214

PROF. H. M. WILIGING, College of St. Thomas, St. Paul, Mn. 55101

DR. WILKINS, Dept. Hd. For. Langs., Lethbridge College Inst., Lethbridge Alberta, Canada

DR. GEORGE W. WILKINS JR., DLL, Tulane Univ., New Orleans, La. 70118

DR. F. H. WILLECKE, Chrm. For. Lang. Dept., Wagner College, Staten Isl., N. Y. 10301

MR. WILLEY, Dir. Fr. Dept., Hyde School, Bath, Me. 04530

CHAS B. WILLIAMS, DLL, State Univ. College, Sheldon Hall, Oswego, N. Y. 13126 
C. A. VON WILIEBRAND, Kauppakorkeakoula, Helsinki Sch. Economics, Helsinki, Finland

MRS. IONE WILLIS, RR 3, Milton, Ky. 40045

DR. ROBERT WILUIS, Eng. Dept., 32 Washington Place, Rm. 32, New York, N. Y. 10013

LIBRARY, Wilton Sr. H. S., Wilton, Ct. 06897

REV. PROF. FRANK M. WINN, Eastern Baptist College, Box 53, St. Davids, Pa. 19087

MRS. T. VADEN WINN, Pearsall H. S., Box 1291, Pearsall, Tx. 78061

WINNEPEG SCHOOL DIV., Languages Dept., 1577 Wall St. East, Winnepeg, 3 Man., Canada

JOE WIPF, 874 Bricker Blvd., Columbus, Ohio 43221

DAVID L. WOHR, 1831 N. Broad St., Philadelphia, Pa. 19122

CHARLES H. WOODFORD, Harper and Row Publishing Inc., 49 East 33rd St., New York, N. Y. 10016

ROBERT W. WOODWORTH, New Haven College, W. Haven, Ct. 06516

DIRECTOR LANGUAGE LAB., The College of Wooster, Wooster, Ohio 44691 MISS CAROLYN WYNN, C-2 Georgetown Village, Spartanburg, S. C. 29301

$$
\text { - Y - }
$$

MRS. J. M. YALDEN, Lect., Carleton Univ., Dept. SP, Ottawa 1 Ontario, Canada

M. L. YEAKEY, Dept. Chmn., Holland Hall School, 2640 S. Birmingham Pl., Tulsa, Ok. 74114

ROBERT YODER, Goshen Col., 1005 South 8th St., Goshen, In. 46526

GEORGE S. YOSHISHIGE, Aud, Vis. Cent., Leeword Comm. Col., 860 Fourth St., Pearl City, Hi. 96782

JOHN N. K. YOUNG, Dir., FL Dept., The MacMillian Co., 866 Third Ave., New York, N. Y. 10022

WM. R. YOUNG, 3019 Monticello, Dallas, Tx. 75205

GEORGE S. YUMICH, Balisdell Pub. Co., 275 Wyman St., Waltham, Ma. 02154

JAMES YZENBAARD, DLL, University of Kentucky, School of Letters and Langs., Lexington, Ky. 40506

$$
-\mathbf{Z}-
$$

VALOIS A. ZARR, Chrm. Ger. Dept., East High School, 1135 South 17th E., Salt Lake City, Ut. 84108

ZEITUNGSVERTRIEBSAMT, Satzabteilung, Clara-Zetkin-Strasse, (1004) Berlin, Germany

PROF. ERNESTO ZIERER, Chrm., Dept. Ed. Idiomas Y Ling., Apt. 315, Univ. NAC, Trujillo, Peru

S. DOROTHY ZIMMERMAN, DLL, Mount Mary College, Milwaukee, Wis. 53222

ELLEN ZIMMERMAN, DLL, Thornridge High School, 150-00 Cottage Grove, Dolton, Il. 60419

DR. MARKO ZUZIC, Chmn., Salem Col., 174 Liberty St., Salem, W. Va. 26426 


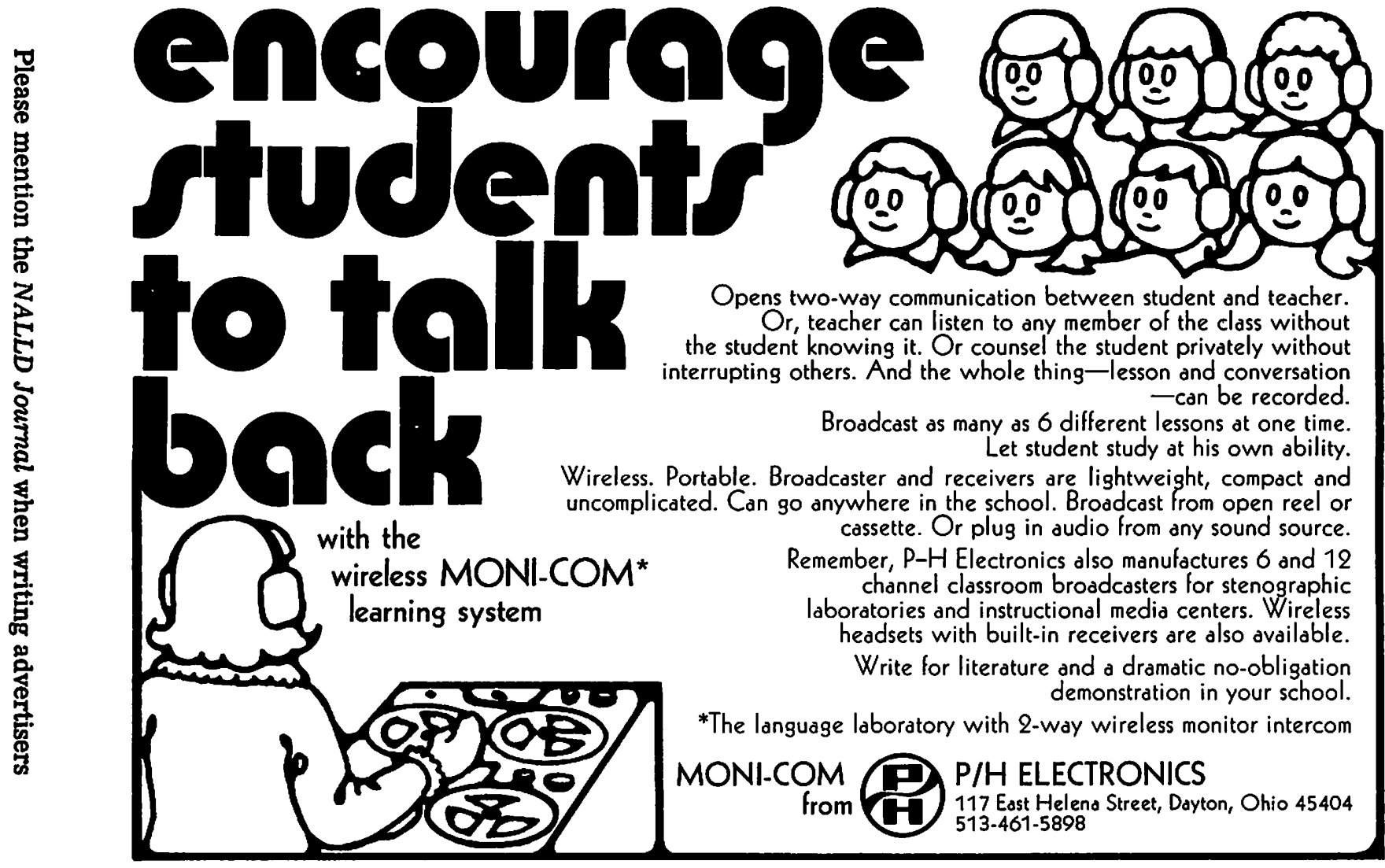

